\title{
On the Calderón-Zygmund Theory for Nonlinear Parabolic Problems with Nonstandard Growth Condition
}

\author{
André H. Erhardt ${ }^{1}$ \\ ${ }^{1}$ Institute for Applied Mathematics and Statistics, University of Hohenheim, Stuttgart, Germany \\ Correspondence: André H. Erhardt, Institute for Applied Mathematics and Statistics, Emil-Wolff-Straße 27, 70599 \\ Stuttgart, Germany. E-mail: andre.erhardt@uni-hohenheim.de
}

Received: November 3, 2014 Accepted: November 21, 2014 Online Published: January 7, 2015

doi:10.5539/jmr.v7n1p10 URL: http://dx.doi.org/10.5539/jmr.v7n1p10

\begin{abstract}
We prove Calderón-Zygmund estimates for a class of parabolic problems whose model is the non-homogeneous parabolic $p(x, t)$-Laplacian equation

$$
\partial_{t} u-\operatorname{div}\left(|D u|^{p(x, t)-2} D u\right)=f-\operatorname{div}\left(|F|^{p(x, t)-2} F\right) .
$$

More precisely, we will show that the spatial gradient $D u$ is as integrable as the inhomogeneities $f$ and $F$, i.e.

$$
|F|^{p(x, t)},|f|^{\frac{\gamma_{1}}{\gamma_{1}-1}} \in L_{\mathrm{loc}}^{q} \Rightarrow|F|^{p(x, t)} \in L_{\text {loc }}^{q} \text { for any } q>1,
$$

where $\gamma_{1}$ is the lower bound for $p(x, t)$. Moreover, it is possible to use this approach to establish the CalderónZygmund theory for parabolic obstacle problems with $p(x, t)$-growth.
\end{abstract}

Keywords: Calderón-Zygmund theory, nonlinear parabolic problem, nonstandard growth

2010 Mathematics Subject Classification: 35K55, 35B45, 35B51, 42B20, 49N60

\section{Introduction}

In this manuscript, we consider nonlinear parabolic equations of the type

$$
\partial_{t} u-\operatorname{div} a(z, D u)=f-\operatorname{div}\left(|F|^{p(z)-2} F\right) \quad \text { in } \quad \Omega_{T}:=\Omega \times(0, T),
$$

where $\Omega \subseteq \mathbb{R}^{n}$ is a bounded domain of dimension $n \geq 2$ and $T>0$ is the height of the space-time cylinder $\Omega_{T}$. Moreover, $\partial_{t} u$ denotes the partial derivative with respect to the time variable $t$, while $D u$ denotes the one with respect to the space variable $x$. Furthermore, we write $z:=(x, t)$ for points in $\Omega_{T}$ and $\partial_{\mathcal{P}} \Omega_{T}=(\bar{\Omega} \times\{0\}) \cup(\partial \Omega \times$ $(0, T)$ ) for the parabolic boundary of $\Omega_{T}$. The vector-field $a(z, D u)$ satisfies certain nonstandard $p(x, t)$-growth and ellipticity conditions which we will note above. First of all, we want to mention the aim of this paper and the importance of nonstandard growth problems. The goal of this paper is to establish local Calderón-Zygmund estimates for $D u$ of solutions to the parabolic problem (1), since these estimates imply that $D u$ is as integrable as the inhomogeneities $f$ and $F$, i.e.

$$
|F|^{p(x, t)},|f|^{\frac{\gamma_{1}}{\gamma_{1}-1}} \in L_{\mathrm{loc}}^{q} \Rightarrow|F|^{p(x, t)} \in L_{\mathrm{loc}}^{q} \text { for any } q>1,
$$

where $\gamma_{1}$ is the lower bound for $p(x, t)$. Moreover, we want to mention that the approach we use here, could also be utilized to establish the Calderón-Zygmund theory for parabolic obstacle problems related to (1). Notice that in (Erhardt, 2014) the Calderón-Zygmund estimates for parabolic obstacle problem related to the model case with $a(z, D u)=a(z)|D u|^{p(z)-2} D u$, where $\mu \leq a(z) \leq L$ satisfies a certain VMO condition, has been already proved. But it is possible to combine the approach of this paper with the one in (Erhardt, 2014) to gain the Calderón-Zygmund estimates for parabolic obstacle problems with irregular obstacles related to the parabolic problem (1).

\subsection{Physical Motivation}

The motivation of considering parabolic partial differential equations is based on the fact that evolutionary equations and systems can be used to model physical processes, e.g. heat conduction or diffusion processes. The 
Navier-Stokes equations, the basic equations of fluid mechanics, is one important example. Also parabolic obstacle problems are motivated by numerous applications, e.g. in mathematical physics, mechanics, control theory or in mathematical biology. For more details and a good overview, we refer to the monograph (Scheven, 2011). Moreover, we want to highlight the importance on nonstandard growth in applications. The following system of a Navier-Stokes equation describes electro-rheological fluids

$$
\left\{\begin{aligned}
-\operatorname{div}\left(\left(1+|\mathcal{E}(u)|^{2}\right)^{\left.\frac{p(x)-2}{2} \mathcal{E}(u)\right)+D \pi}\right. & =f(x, u, D u), \\
\operatorname{div}(u) & =0
\end{aligned}\right.
$$

where $\mathcal{E}(u)$ is the symmetric part of the gradient $D u, \pi$ denotes the pressure and the variable growth exponent $p(x)$ is a Hölder continuous function. Such fluids are of high technological interest because of their ability to change the mechanical properties under the influence of exterior electro-magnetic field, cf. (Ettwein \& Růǔička, 2003), (Růǔička, 2000). For example, many electro-rheological fluids are suspensions consisting of solid particles and a carrier oil. These suspensions change their material properties dramatically if they are exposed to an electric field, see (Růǔička, 2004). Most of the known results concern the stationary models, see for example (Acerbi \& Mingione, $2001 \& 2002 \mathrm{a}, \mathrm{b})$. Moreover, the non-stationary case, i.e. the model depending on variable exponents $p(x, t)$ has been studied in (Acerbi, Mingione \& Seregin, 2004). Here, we are going to study similar parabolic problems with $p(x, t)$-growth and we will establish a further regularity result. Other applications are the models for flows in porous media, see (Antontsev \& Shmarev, 2005).

\subsection{General Assumptions}

First of all, we shall consider vector-fields $a: \Omega_{T} \times \mathbb{R}^{n} \rightarrow \mathbb{R}^{n}$ which are assumed to be Carathéodory functions i.e. $a(z, w)$ is measurable in the first argument for every $w \in \mathbb{R}^{n}$ and continuous in the second one for a.e. $z \in \Omega_{T}$ - and satisfy the following nonstandard growth, monotonicity and ellipticity conditions for some growth exponent function $p: \Omega_{T} \rightarrow\left(\frac{2 n}{n+2}, \infty\right)$ and structure constants $0<\mu \leq 1<L$ and $s \in[0,1]$ :

$$
\begin{aligned}
|a(z, w)| & \leq L(1+|w|)^{p(z)-1}, \\
\left(a(z, w)-a\left(z, w_{0}\right)\right) \cdot\left(w-w_{0}\right) & \geq \mu\left(s^{2}+|w|^{2}+\left|w_{0}\right|^{2}\right)^{\frac{p(z)-2}{2}}\left|w-w_{0}\right|^{2}, \\
\left|D_{w} a(z, w)\right| & \leq L(1+|w|)^{p(z)-2} \\
D_{w} a(z, w) \xi \cdot \xi & \geq \mu\left(s^{2}+|w|^{2}\right)^{\frac{p(z)-2}{2}}
\end{aligned}
$$

for all $z \in \Omega_{T}$ and $w, w_{0}, \xi \in \mathbb{R}^{n}$. Moreover, there exist constants $\gamma_{1}, \gamma_{2}<\infty$ such that the growth exponent $p: \Omega_{T} \rightarrow\left(\frac{2 n}{n+2}, \infty\right)$ satisfies

$$
\frac{2 n}{n+2}<\gamma_{1} \leq p(z) \leq \gamma_{2} \text { and }\left|p\left(z_{1}\right)-p\left(z_{2}\right)\right| \leq \omega\left(d_{\mathcal{P}}\left(z_{1}, z_{2}\right)\right)
$$

for any $z_{1}=\left(x_{1}, t_{1}\right), z_{2}=\left(x_{2}, t_{2}\right) \in \Omega_{T}$, where $\omega:[0, \infty) \rightarrow[0,1]$ denotes a modulus of continuity which is assumed to be concave and non-decreasing with $\lim _{\varrho \downarrow 0} \omega(\varrho)=0=\omega(0)$. The parabolic distance is given by $d_{\mathcal{P}}\left(z_{1}, z_{2}\right):=\max \left\{\left|x_{1}-x_{2}\right|, \sqrt{\left|t_{1}-t_{2}\right|}\right\}$ for $z_{1}, z_{2} \in \Omega_{T}$. In addition, for $\omega(\cdot)$ we assume that

$$
\underset{\varrho \downarrow 0}{\lim \sup } \omega(\varrho) \log \left(\frac{1}{\varrho}\right)=0 .
$$

By virtue of (7) we may assume that there exists $\mathfrak{R} \in(0,1]$ depending on $\omega(\cdot)$ such that

$$
\omega(\varrho) \log \left(\frac{1}{\varrho}\right) \leq 1 \text { for all } \varrho \in(0, \Re]
$$

Here, we want to mention that the monotonicity property (3) is a consequence of the ellipticity condition (5). Finally, we suppose the following continuity assumption

$$
\left|a\left(z_{1}, w\right)-a\left(z_{2}, w\right)\right| \leq \sqrt{L} \omega\left(d_{\mathcal{P}}\left(z_{1}, z_{2}\right)\right)\left[(1+|w|)^{p\left(z_{1}\right)-1}+(1+|w|)^{p\left(z_{2}\right)-1}\right] \cdot[1+\log (1+|w|)]
$$

for all $z_{1}, z_{2} \in \Omega_{T}$ and $w \in \mathbb{R}^{n}$.

\subsection{Historical Background - a Short Overview}


The history of the Calderón-Zygmund theory for nonlinear problems starts in the elliptic case. The result for the stationary $p$-Laplacian equation was established in (Iwaniec, 1983), while the result for the $p$-Laplacian system was proved in (DiBenedetto \& Manfredi, 1993). The fact, that for elliptic equations with VMO coefficients, the Calderón-Zygmund estimates for the gradient on $L^{q}$-level for $q>p$ are valid, was shown in (Kinnunen \& Zhou, 1999). Notice that, VMO-type conditions are very weak assumptions on the regularity of the coefficients. General elliptic equations, also involving nonstandard growth conditions, have been treated in (Acerbi \& Mingione, 2005) who built on previous ideas of Caffarelli and Peral in (Cafferelli \& Peral, 1998) valid for homogeneous equations with highly oscillating coefficients. For the case of higher order systems with nonstandard $p(x)$-growth conditions we refer to (Habermann, 2008). In (Eleuteri \& Habermann, 2010) some Calderón-Zygmund results for equations and systems with nonstandard growth conditions, mainly for obstacle problems with $p(x)$-growth, were established. The result for the parabolic evolutionary p-Laplacian system has finally been achieved by Acerbi and Mingione in (Acerbi \& Mingione, 2007) who introduced the necessary new tools for developing a local Calderón-Zygmund theory for the time dependent, parabolic case, see also (Misawa, 2005) for the special case $F \in$ BMO. Later on, extensions to general parabolic systems have been obtained in (Duzaar, Mingione \& Steffen, 2011), see also (Scheven, 2010). Moreover, a Calderón-Zygmund theory for obstacle problems was first established in (Bögelein, Duzaar \& Mingione, 2011) and then, Scheven extends this result to obstacle problems with VMO-coefficients in (Scheven, 2011 \& 2014). Furthermore, the Calderón-Zygmund theory for elliptic and parabolic measure data equations are proved in (Mingione, 2007a,b) and (Baroni \& Habermann, 2012), respectively. The global gradient estimates for degenerate and singular parabolic systems are again established in (Bögelein, 2013).

In the context of nonstandard $p(x, t)$-growth we want to mention that the Calderón-Zygmund theory for parabolic $p(x, t)$-Laplacian systems was shown in (Baroni \& Bögelein, 2013), see also the monograph (Baroni, 2013), while the Calderón-Zygmund theory for parabolic obstacle problems related to the parabolic $p(x, t)$-Laplacian was established in (Erhardt, 2014), see also the monograph (Erhardt, 2013). As we already mentioned above the approach we use here, could also applied to parabolic obstacle problems with $p(x, t)$-growth. Therefore, we are able to prove the result in (Erhardt, 2014) for parabolic obstacle problem with irregular obstacles related to (1).

\subsection{The Function Spaces}

The spaces $L^{p}(\Omega), W^{1, p}(\Omega)$ and $W_{0}^{1, p}(\Omega)$ stand for the usual Lebesgue and Sobolev spaces.

Parabolic Lebesgue-Orlicz spaces. We start by the definition of the nonstandard $p(z)$-Lebesgue space. The space $L^{p(z)}\left(\Omega_{T}, \mathbb{R}^{k}\right)$ is defined as the set of those measurable functions $v: \Omega_{T} \rightarrow \mathbb{R}^{k}$ for $k \in \mathbb{N}$, such that $|v|^{p(\cdot)} \in$ $L^{1}\left(\Omega_{T}, \mathbb{R}^{k}\right)$, i.e.

$$
L^{p(z)}\left(\Omega_{T}, \mathbb{R}^{k}\right):=\left\{v: \Omega_{T} \rightarrow \mathbb{R}^{k} \text { is measurable in } \Omega_{T}: \int_{\Omega_{T}}|v|^{p(\cdot)} \mathrm{d} z<+\infty\right\} .
$$

The set $L^{p(\cdot)}\left(\Omega_{T}, \mathbb{R}^{k}\right)$ equipped with the Luxemburg norm

$$
\|v\|_{L^{p()}\left(\Omega_{T}\right)}:=\inf \left\{\lambda>0: \int_{\Omega_{T}}\left|\frac{v}{\lambda}\right|^{p(z)} \mathrm{d} z \leq 1\right\}
$$

becomes a Banach space.

Parabolic Sobolev-Orlicz spaces. Next, we introduce nonstandard parabolic Sobolev spaces. By $W_{g}^{p(\cdot)}\left(\Omega_{T}\right)$ we denote the Banach space

$$
W_{g}^{p(\cdot)}\left(\Omega_{T}\right):=\left\{u \in\left[g+L^{1}\left(0, T ; W_{0}^{1,1}(\Omega)\right)\right] \cap L^{p(\cdot)}\left(\Omega_{T}\right) \mid D u \in L^{p(\cdot)}\left(\Omega_{T}, \mathbb{R}^{n}\right)\right\}
$$

equipped by the norm $\|u\|_{W^{p()}\left(\Omega_{T}\right)}:=\|u\|_{L^{p(\cdot)}\left(\Omega_{T}\right)}+\|D u\|_{L^{(\cdot)}\left(\Omega_{T}\right)}$. If $g=0$ we write $W_{0}^{p(\cdot)}\left(\Omega_{T}\right)$ instead of $W_{g}^{p(\cdot)}\left(\Omega_{T}\right)$. Finally, we shall assume that

$$
F \in L^{p(\cdot)}\left(\Omega_{T}, \mathbb{R}^{n}\right) \text { and } f \in L^{\gamma_{1}^{\prime}}\left(\Omega_{T}\right) .
$$

Definition 1. We identify a function $u \in L^{1}\left(\Omega_{T}\right)$ as a weak solution of the parabolic equation (1), if and only if $u \in C^{0}\left([0, T] ; L^{2}(\Omega)\right) \cap W^{p(\cdot)}\left(\Omega_{T}\right)$ and

$$
\int_{\Omega_{T}}\left[u \cdot \varphi_{t}-|D u|^{p(\cdot)-2} D u \cdot D \varphi\right] \mathrm{d} z=-\int_{\Omega_{T}}\left[f \cdot \varphi+|F|^{p(\cdot)-2} F \cdot D \varphi\right] \mathrm{d} z
$$

holds, whenever $\varphi \in C_{0}^{\infty}\left(\Omega_{T}\right)$. 
Notice that the existence of a weak solution of (1) is guaranteed by the result in (Erhardt, 2013b), see also (Erhardt, 2013a).

Our next aim is to introduce the dual space of $W_{0}^{p(\cdot)}\left(\Omega_{T}\right)$. Therefore, we denote by $W^{p(\cdot)}\left(\Omega_{T}\right)^{\prime}$ the dual of $W_{0}^{p(\cdot)}\left(\Omega_{T}\right)$. Here and in the following, we write $\langle\langle\cdot, \cdot\rangle\rangle_{\Omega_{T}}$ for the pairing between $W^{p(\cdot)}\left(\Omega_{T}\right)^{\prime}$ and $W_{0}^{p(\cdot)}\left(\Omega_{T}\right)$, see also (Erhardt, 2013a). Furthermore, if $v \in W^{p(\cdot)}\left(\Omega_{T}\right)^{\prime}$, we define the norm $\|v\|_{W^{p(\cdot)}\left(\Omega_{T}\right)^{\prime}}=\sup \left\{\langle v, w\rangle \Omega_{\Omega_{T}} \mid w \in\right.$ $\left.W_{0}^{p(\cdot)}\left(\Omega_{T}\right),\|w\|_{W_{0}^{p(\cdot)}\left(\Omega_{T}\right)} \leq 1\right\}$. By $w \in W\left(\Omega_{T}\right):=\left\{w \in W_{0}^{p(\cdot)}\left(\Omega_{T}\right) \mid w_{t} \in W^{p(\cdot)}\left(\Omega_{T}\right)^{\prime}\right\}$ we mean that there exists $w_{t} \in W^{p(\cdot)}\left(\Omega_{T}\right)^{\prime}$, such that

$$
\left\langle\left\langle w_{t}, \varphi\right\rangle\right\rangle_{\Omega_{T}}=-\int_{\Omega_{T}} w \cdot \varphi_{t} \mathrm{~d} z \text { for all } \varphi \in C_{0}^{\infty}\left(\Omega_{T}\right) .
$$

The previous equality makes sense due to the inclusions $W^{p(\cdot)}\left(\Omega_{T}\right) \hookrightarrow L^{2}\left(\Omega_{T}\right) \cong\left(L^{2}\left(\Omega_{T}\right)\right)^{\prime} \hookrightarrow W^{p(\cdot)}\left(\Omega_{T}\right)^{\prime}$ which allow us to identify $w$ as an element of $W^{p(\cdot)}\left(\Omega_{T}\right)^{\prime}$. As a consequence of this embedding, functions $w \in W\left(\Omega_{T}\right)$ that vanish on the lateral boundary also satisfy $C^{0}\left([0, T] ; L^{2}(\Omega)\right)$. More precisely, we refer the following lemma which is established in (Erhardt, 2013a,b).

Lemma 2. Let $n \geq 2$. Assume that the exponent function $p: \Omega_{T} \rightarrow\left[\gamma_{1}, \gamma_{2}\right]$ satisfies (6)-(8). Then W( $\left.\Omega_{T}\right)$ is contained in $C^{0}\left([0, T] ; L^{2}(\Omega)\right)$. Moreover, if $u \in W\left(\Omega_{T}\right)$ then $t \mapsto\|u(\cdot, t)\|_{L^{2}(\Omega)}^{2}$ is absolutely continuous on $[0, T]$,

$$
\frac{d}{d t} \int_{\Omega}|u(\cdot, t)|^{2} d x=2\left\langle\partial_{t} u(\cdot, t), u(\cdot, t)\right\rangle, \text { for a.e. } t \in[0, T],
$$

where $\langle\cdot, \cdot\rangle$ denotes the duality pairing between $W^{1, p(\cdot, t)}(\Omega)^{\prime}$ and $W_{0}^{1, p(\cdot, t)}(\Omega)$. Moreover, there is a constant $c$ for which $\|u\|_{C^{0}\left([0, T] ; L^{2}(\Omega)\right)} \leq c\|u\|_{W\left(\Omega_{T}\right)}$ holds for every $u \in W\left(\Omega_{T}\right)$.

\subsection{Intrinsic Geometry}

Before we are able to state the result, we have to mention a very important concept in the parabolic regularity theory. Therefore, we introduce symmetric parabolic cylinders with center in $z_{0}=\left(x_{0}, t_{0}\right) \in \Omega_{T}$ of the form $Q_{\varrho}\left(z_{0}\right):=B_{\varrho}\left(x_{0}\right) \times\left(t_{0}-\varrho^{2}, t_{0}+\varrho^{2}\right)$, where $\left(t_{0}-\varrho^{2}, t_{0}+\varrho^{2}\right) \subset(0, T)$ and $B_{\varrho}\left(x_{0}\right) \subset \Omega$ denotes a ball with radius $\varrho>0$ and center $x_{0}$. To obtain the relevant (scaling invariant) local estimates we will use, in order to re-balance the non-homogeneity of parabolic problems, certain scaled cylinders, i.e. so-called intrinsic cylinders of the form

$$
Q_{\varrho}^{(\lambda)}\left(z_{0}\right):=B_{\varrho}\left(x_{0}\right) \times \Lambda_{\varrho}^{(\lambda)}\left(t_{0}\right), \text { where } \Lambda_{\varrho}^{(\lambda)}\left(t_{0}\right):=\left(t_{0}-\lambda^{\frac{2-p_{0}}{p_{0}}} \varrho^{2}, t_{0}+\lambda^{\frac{2-p_{0}}{p_{0}}} \varrho^{2}\right),
$$

where $\lambda>0$ and $p_{0}:=p\left(z_{0}\right)$. The reason for such scaled cylinder is based on the fact (explained by the easiest problem), that a multiple $c \cdot u$ of a solution to $\partial_{t} u-\operatorname{div}\left(|D u|^{p-2} D u\right)=0$ is no longer a solution, except $c \in\{0,1\}$, $p=2$ or $u \equiv 0$. Such kind of intrinsic cylinders were introduced in the case $p=$ const. in the pioneering work of DiBenedetto and Friedman in (DiBenedetto \& Friedman, 1985a,b). The way we use the idea of intrinsic cylinders goes back to Bögelein and Duzaar in (Bögelein \& Duzaar, 2012). The delicate aspect in this technique relies in the fact that the cylinders will be constructed in such a way, that the scaling parameter $\lambda>0$ and the average of $|D u|^{p(\cdot)}$ over $Q_{\varrho}^{(\lambda)}\left(z_{0}\right)$ are coupled in the following way:

$$
\int_{Q_{\varrho}^{(\lambda)}\left(z_{0}\right)}|D u|^{p(\cdot)} \mathrm{d} z \approx \lambda
$$

\section{Strategy of the Proof and Preliminary Results}

In this section, we establish the result and describe the strategy of the proof.

\subsection{The statement and the strategy of the proof}

First of all, we state the main result of this paper.

Theorem 3. Assume that $p: \Omega_{T} \rightarrow\left[\gamma_{1}, \gamma_{2}\right]$ satisfies (6)-(8) and the assumptions (2)-(5) and (9) on the vector-field $a: \Omega_{T} \times \mathbb{R}^{n} \rightarrow \mathbb{R}^{n}$ are valid. Moreover, the inhomogeneities (10) are given. Additionally, we suppose that the data has the higher integrability properties

$$
|F|^{p(\cdot)} \in L_{l o c}^{q}\left(\Omega_{T}\right) \text { and }|f|^{\gamma_{1}^{\prime}} \in L_{l o c}^{q}\left(\Omega_{T}\right) \text { for some } q>1 .
$$


Then, every weak solution $u \in C^{0}\left([0, T] ; L^{2}(\Omega)\right) \cap W^{p(\cdot)}\left(\Omega_{T}\right)$ of the parabolic equation (1) satisfies

$$
|D u|^{p(\cdot)} \in L_{l o c}^{q}\left(\Omega_{T}\right) .
$$

Moreover, for $\kappa, K \geq 1$ there exists a radius $r_{0}>0$ depending on $n, \gamma_{1}, \gamma_{2}, q, \mu, L, \sigma, \kappa, K, \omega(\cdot)$ and a constant $c=c\left(n, \gamma_{1}, \gamma_{2}, q, \mu, L, \sigma, \kappa, K, \omega(\cdot)\right)$, such that the following holds: If

$$
\int_{\Omega_{T}}\left(|D u|^{p(\cdot)}+|F|^{p(\cdot)}+|f|^{\gamma_{1}^{\prime}}+1\right) \mathrm{d} z \leq K
$$

is satisfied, then for every parabolic cylinder $Q_{2 r} \equiv Q_{2 r}\left(\mathfrak{z}_{0}\right) \Subset \Omega_{T}$ with radius $r \in\left(0, r_{0}\right]$, there holds

$$
\int_{Q_{r}}|D u|^{p(\cdot) q} \mathrm{~d} z \leq c\left[f_{Q_{2 r}}|D u|^{p(\cdot)} \mathrm{d} z\left(f_{Q_{2 r}}\left(|F|^{p(\cdot)}+|f|^{\gamma_{1}^{\prime}}+1\right)^{q} \mathrm{~d} z\right)^{\frac{1}{q}}\right]^{1+d\left(p_{0}\right)(q-1)},
$$

where $p_{0}=p\left(\mathfrak{z}_{0}\right)$ and $d\left(p_{0}\right)$ is defined in

$$
\begin{cases}\frac{2 p_{0}}{p_{0}(n+2)-2 n} & \text { if } p_{0}<2 \\ \frac{p_{0}}{2} & \text { if } p_{0} \geq 2 .\end{cases}
$$

Now, we briefly describe the plan of the paper and the strategy of the proof. As we mentioned in the introduction the desired result of this manuscript is to prove the gradient estimate (13). This estimate implies that $D u$ is as integrable as the inhomogeneities $F$ and $f$, cf. Theorem 3. For the proof of the gradient estimate we will use some comparison arguments to derive the needed a priori estimate. In Section 2.3, we will compare the solution of (1) with the solution of a certain homogeneous parabolic Cauchy-Dirichlet problem with nonstandard growth, then we will compare the solution of the Cauchy-Dirichlet problem with the solution of a parabolic problem with frozen exponent, i.e. with exponent $p_{0}=p\left(z_{0}\right)$. Moreover, we will need several technical tools to gain the desired gradient estimate, e.g. the higher integrability result of Bögelein and Duzaar, cf. Theorem 6, a localization argument of Baroni and Bögelein, cf. Lemma 7, and the Lipschitz bound, which is a consequence of the $C^{1, \alpha}$-regularity of DiBenedetto and Friedman, cf. Theorem 9. Later on, we will transfer these a priori estimates via comparison argument to our nonstandard growth problem. All these preliminary results and many more are stated in Section 2.2. Finally, in Section 3 we will prove the main result. Here, we start with a so-called stopping time argument. This argument is very important in the regularity of parabolic problems, since there we will choose the correct cylinders, cf. (Kinnunen \& Lewis, 2000) and (Acerbi \& Mingione, 2005) for instance. Then, we will apply the comparison estimates of Section 2.3 on certain intrinsic cylinders. The next step is to derive estimates on the level sets, followed by the final estimate, which is in principle the desired gradient estimate. The final step of the proof of Theorem 3 is to adjust the exponent. In this step we will conclude the validity of (13).

\subsection{Technical Tools and Preliminary Results}

In this section, we cite several important technical tools, which we need for the proof of the desired CalderónZygmund estimates. Moreover, we will prove some tools which are important to derive our main result.

An iteration lemma. In order to "re-absorb" certain terms, we will use the following iteration lemma, which is a standard tool and can be found in (Giaquinta, 1983). The iteration results reads as follows.

Lemma 4. Let $0<\vartheta<1, A, C \geq 0$ and $\beta>0$. Then, there exists a constant $c=c(\beta, \vartheta)$, such that there holds: For any non-negative bounded function satisfying $\Phi(t) \leq \vartheta \Phi(s)+A(s-t)^{-\beta}+C$ for all $0<r \leq t<s \leq \varrho$, we have

$$
\Phi(r) \leq c\left[A(\varrho-r)^{-\beta}+C\right]
$$

A version of the Vitali's covering Theorem. Moreover, we will need a version of the Vitali's covering Theorem for non-uniformly intrinsic parabolic cylinders, which is stated in (Bögelein \& Duzaar, 2011). The result is the following:

Lemma 5. Assume that $M \geq 1, \lambda \geq 1$ and $p: \Omega_{T} \rightarrow\left(\gamma_{1}, \gamma_{2}\right)$ satisfies the conditions (6)-(8). Then, there exists a constant $\chi=\chi\left(n, L_{1}, \gamma_{1}\right) \geq 5$, such that the following is true: Let $\mathcal{F}=\left\{Q_{i}\right\}_{i \in I}$ be a family of axially parallel 
parabolic cylinders of the from $Q_{i}=Q_{\varrho_{i}}^{(\lambda)}\left(z_{i}\right):=B_{\varrho_{i}}\left(x_{i}\right) \times\left(t_{i}-\lambda^{\frac{2-p\left(z_{i}\right)}{p\left(z_{i}\right)}} \varrho_{i}^{2}, t_{i}+\lambda^{\frac{2-p\left(z_{i}\right)}{p\left(z_{i}\right)}} \varrho_{i}^{2}\right)$ with uniformly bounded radii, uniformly in the sense that

$$
\varrho_{i} \leq \min \left\{\varrho_{0},\left[\left(\beta_{n} M\right)^{\frac{p\left(z_{i}\right)}{2}} \lambda^{-1}\right]^{\frac{2}{p\left(z_{i}\right)(n+2)}}\right\} \quad \forall i \in \mathcal{I}
$$

where $\varrho_{0}:=\left[6 \sqrt{\beta_{n} M}\right]^{-\frac{2}{\alpha}} \leq 1$. Then, there exists a countable sub-collection $\mathcal{G} \subseteq \mathcal{F}$ of disjoint parabolic cylinders, such that $\bigcup_{Q \in \mathcal{F}} Q \subset \bigcup_{Q \in \mathcal{G}} \chi Q$, where $\chi Q$ denotes the $\chi$-time enlarged cylinder $Q$, i.e. if $Q=Q_{\varrho}^{(\lambda)}(z)$ then $\chi Q=Q_{\chi \varrho}^{(\lambda)}(z)$.

Iwaniec's inequality. Here, we state a useful estimate which is a consequence of Iwaniec's inequality for Orlicz spaces, see (Iwaniec \& Verde, 1999). Let $\vartheta>0, Q \subset \mathbb{R}^{n+1}$ and $\mathfrak{g} \in L^{\varsigma}(Q)$ for some $\varsigma>1$. Then, there holds

$$
f_{Q}|\mathfrak{g}| \log ^{\sigma}\left(e+\frac{|\mathfrak{g}|}{(\mathfrak{g})_{Q}}\right) \mathrm{d} z \leq c(\vartheta, \varsigma)\left(f_{Q}|\mathfrak{g}|^{\varsigma} \mathrm{d} z\right)^{\frac{1}{\varsigma}} \quad \text { for all } \varsigma>1 .
$$

Thereby, the constant $c(\vartheta, \varsigma)$ blows up as $\varsigma \downarrow 1$. Moreover, $c(\vartheta, \varsigma)$ depends continuously on $\vartheta$ and therefore, it can be replaced by a constant $c\left(\gamma_{1}, \gamma_{2}, \varsigma\right)$ if $\vartheta \in\left[\gamma_{2}^{\prime}, \gamma_{1}^{\prime}\right]$.

A higher integrability estimate. The higher integrability result will play a key role in the proof of the CalderónZygmund estimates. In the case $p(\cdot)=$ const. the needed higher integrability estimate goes back to Kinnunen and Lewis (Kinnunen \& Lewis, 2000). In the following, we cite higher integrability estimate for degenerate parabolic equations with nonstandard growth from (Erhardt, 2013c), which is a little modification of the result in (Bögelein \& Duzaar, 2011), because they considered parabolic problem of the form

$$
\partial_{t} u-\operatorname{div} a(z, D u)=\operatorname{div}\left(|F|^{p(\cdot)-2} F\right) \quad \text { in } \Omega_{T}
$$

instead of (1). Notice that this modification is a simple exercise. The statement reads as follows:

Theorem 6. Let $\sigma>0$ and $p: \Omega_{T} \rightarrow\left[\gamma_{1}, \gamma_{2}\right]$ satisfy (6)-(8). Then, there exists $\varepsilon_{0}=\varepsilon_{0}\left(n, \gamma_{1}, \gamma_{2}, \mu, L, L_{1}, \sigma\right) \in$ $(0, \sigma]$, such that the following holds: Whenever a function $u \in C^{0}\left([0, T] ; L^{2}(\Omega)\right) \cap W^{p(\cdot)}\left(\Omega_{T}\right)$ is a weak solution of the parabolic equation (1), where (2)-(3) are in force and $f \in L^{\gamma_{1}^{\prime}(1+\sigma)}\left(\Omega_{T}\right), F \in L^{p(\cdot)(1+\sigma)}\left(\Omega_{T}\right)$, then

$$
|D u|^{p(\cdot)\left(1+\varepsilon_{0}\right)} \in L_{l o c}^{1}\left(\Omega_{T}\right) \text {. }
$$

Moreover, for any $K \geq 1$ there exists a radius $\varrho_{1} \equiv \varrho_{1}\left(n, \gamma_{1}, \gamma_{2}, L_{1}, K, \omega(\cdot)\right)>0$, such that there holds: If (12) and $\varepsilon \in\left(0, \varepsilon_{0}\right]$, then for any parabolic cylinder $Q_{2 r} \equiv Q_{2 r}\left(\mathfrak{z}_{0}\right) \subseteq \Omega_{T}$ with $r \in\left(0, \varrho_{1}\right]$, there holds

$$
f_{Q_{r}}|D u|^{p(\cdot)(1+\varepsilon)} \mathrm{d} z \leq c\left(f_{Q_{2 r}}\left(|D u|^{p(\cdot)}+|F|^{p(\cdot)}+|f|^{\gamma_{1}^{\prime}}+1\right) \mathrm{d} z\right)^{1+\varepsilon d}+c f_{Q_{2 r}}\left(|F|^{p(\cdot)}+|f|^{\gamma_{1}^{\prime}}+1\right)^{(1+\varepsilon)} \mathrm{d} z
$$

for a constant $c=c\left(n, \gamma_{1}, \gamma_{2}, \mu, L, L_{1}\right)$, where $d$ is defined in (14).

To apply this result, we need a further technical tool, more precisely a non uniform intrinsic geometry argument. This we will mention in the following lemma, where we provide a parabolic localization technique. This lemma goes back to (Baroni \& Bögelein, 2013). Obviously the difficulty stems from the necessity to couple the technique of intrinsic geometry with the localization needed to treat the variable exponent growth conditions.

Lemma 7. Let $\kappa, K, H \geq 1$ and $p: \Omega_{T} \rightarrow\left[\gamma_{1}, \gamma_{2}\right]$ satisfy (6) and (8). Then, there exists a radius $\varrho_{2}=$ $\varrho_{2}\left(n, \gamma_{1}, \gamma_{2}, \kappa, K, H, \omega(\cdot)\right) \in\left(0, R_{1}\right]$, such that the following holds: Whenever, Du, $F \in L^{p(\cdot)}\left(\Omega_{T}, \mathbb{R}^{n}\right)$ and $f \in$ $L^{\gamma_{1}^{\prime}}\left(\Omega_{T}\right)$ satisfy $(12)$ and $Q_{\varrho}^{(\lambda)}\left(z_{0}\right) \subset \Omega_{T}$ is a parabolic cylinder with $\varrho \in\left(0, \varrho_{2}\right]$ and $\lambda \geq 1$, such that

$$
\lambda \leq \kappa f_{Q_{Q}^{(\lambda)}\left(z_{0}\right)}|D u|^{p(\cdot)}+H\left(|F|^{p(\cdot)}+|f|^{\gamma_{1}^{\prime}}+1\right) \mathrm{d} z
$$

Then, we have

$$
\lambda \leq\left(\frac{\tilde{\Gamma}}{4 \varrho^{n+2}}\right)^{\frac{p_{0}}{2}}, \quad p_{2}-p_{1} \leq \omega\left(\tilde{\Gamma} \varrho^{\alpha}\right) \quad \text { and } \quad \lambda^{\omega\left(\tilde{\Gamma} \varrho^{\alpha}\right)} \leq e^{\frac{3 n L_{1} p_{0}}{\alpha}},
$$


where $p_{0}:=p\left(z_{0}\right), p_{1}:=\inf _{Q_{\varrho}^{(\lambda)}\left(z_{0}\right)} p(\cdot)$ and $p_{2}:=\sup _{Q_{\varrho}^{(\lambda)}\left(z_{0}\right)} p(\cdot)$ and

$$
\tilde{\Gamma}:=4 \beta_{n} \kappa K H, \quad \beta_{n}:=\max \left\{1,\left(2 \alpha_{n}\right)^{-1}\right\}, \quad \alpha:=\min \left\{1, \gamma_{1} \frac{n+2}{4}-\frac{n}{2}\right\} \text {. }
$$

Our next aim is to adapt the local estimate from Theorem 6 to intrinsic cylinders of the form $Q_{\varrho}^{(\lambda)}\left(z_{0}\right):=B_{\varrho}\left(x_{0}\right) \times$ $\Lambda_{\varrho}^{(\lambda)}\left(t_{0}\right)$ for $z_{0}=\left(x_{0}, t_{0}\right) \in \Omega_{T}, \varrho>0$ and $\lambda \geq 1$, where $\Lambda_{\varrho}^{(\lambda)}\left(t_{0}\right):=\left(t_{0}-\lambda^{\frac{2-p_{0}}{p_{0}}} \varrho^{2}, t_{0}+\lambda^{\frac{2-p_{0}}{p_{0}}} \varrho^{2}\right)$. We will determine the parameter $\lambda \geq 1$ in dependence on the solution itself and we will use this intrinsic coupling to compensate the anisotropic scaling behaviour of the parabolic inequality. On cylinders of this type, the higher integrability estimate from the above theorem takes the following from.

Corollary 8. Let $K, c_{*}, \hat{c} \geq 1$ and $\sigma>0$. Assume that the vector field a $: \Omega_{T} \times \mathbb{R}^{n} \rightarrow \mathbb{R}^{n}$ satisfies (2)-(3) and $p: \Omega_{T} \rightarrow \mathbb{R}$ fulfills (6) and (7). Further, suppose that $f \in L^{p(\cdot)^{\prime}(1+\sigma)}\left(\Omega_{T}\right), F \in L^{p(\cdot)(1+\sigma)}\left(\Omega_{T}\right)$ are in force and for $z_{0} \in \Omega_{T}, \varrho \in\left(0, \varrho_{1}\right]$ and $\lambda \geq 1$ with $Q_{2 \varrho}^{(\lambda)}\left(z_{0}\right) \subset \Omega_{T}$, satisfying

$$
\lambda^{p_{2}-p_{1}} \leq \hat{c}, \quad \text { where } p_{1}:=\inf _{Q_{2 \varrho}^{(\lambda)}\left(z_{0}\right)} p(\cdot) \text { and } p_{2}:=\sup _{Q_{2 \varrho}^{(\lambda)}\left(z_{0}\right)} p(\cdot) .
$$

Then, there exists $\varepsilon_{0}=\varepsilon_{0}\left(n, \gamma_{1}, \gamma_{2}, \mu, L, \sigma\right) \in(0, \sigma]$ such that the following holds: Whenever

$$
v \in C^{0}\left(\Lambda_{2 \varrho}^{(\lambda)}\left(t_{0}\right) ; L^{2}\left(B_{2 \varrho}\right)\left(x_{0}\right)\right) \cap W^{p(\cdot)}\left(Q_{2 \varrho}^{(\lambda)}\left(z_{0}\right)\right)
$$

is a weak solution of

$$
\partial_{t} v-\operatorname{div} a(z, D v)=f-\operatorname{div}|F|^{p(\cdot)-2} F \quad \text { on } Q_{2 \varrho}^{(\lambda)}\left(z_{0}\right)
$$

satisfying (12) (with Du replaced by Dv) and

$$
\int_{Q_{2 \varrho}^{(\lambda)}\left(z_{0}\right)}|D v|^{p(\cdot)} \mathrm{d} z \leq c_{*} \lambda
$$

Then, there holds the higher integrability estimate

$$
\int_{Q_{\varrho}^{(\lambda)}\left(z_{0}\right)}|D v|^{p(\cdot)\left(1+\varepsilon_{0}\right)} \mathrm{d} z \leq c \lambda^{1+\varepsilon_{0}}
$$

with a constant $c \geq 1$ depending only on $n, \gamma_{1}, \gamma_{2}, \mu, L, K, c_{*}$ and $\hat{c}$.

Notice that there is a similar result is given by (Baroni \& Bögelein, 2013). The main difference is, that they consider the parabolic system $\partial_{t} v-\operatorname{div}\left(a(z)|D v|^{p(\cdot)-2} D v\right)=0$, where $a: \Omega_{T} \rightarrow \mathbb{R}$ is a measurable function with $\mu \leq a(z) \leq L$ for any $z \in \Omega_{T}$, while we consider again the equation (1). Furthermore, in the standard growth case, there is also as similar result given in (Scheven, 2014).

Proof. First, we assume that $z_{0}=0$. Then, we rescale the problem from intrinsic cylinders $Q_{\varrho}^{(\lambda)}, Q_{2 \varrho}^{(\lambda)}$ to the standard parabolic cylinders $Q_{\varrho}, Q_{2 \varrho}$. Therefore, we have to transform in time and then, we could apply the Theorem 6. Here, we have to discuss two cases. On the one hand the case $p_{0}:=p(0) \geq 2$ and on the other hand $p_{0}:=p(0)<2$. We start with the case $p_{0}:=p(0) \geq 2$ and define for $(x, t) \in Q_{2 \varrho}$ the rescaled exponent $\tilde{p}(x, t)=p\left(x, \lambda^{\frac{2-p_{0}}{p_{0}}} t\right)$. Furthermore, we consider the rescaled function $\tilde{v}(x, t):=\lambda^{-\frac{1}{p_{0}}} v\left(x, \lambda^{\frac{2-p_{0}}{p_{0}}} t\right)$, which implies $D \tilde{v}(x, t)=\lambda^{-\frac{1}{p_{0}}} D v\left(x, \lambda^{\frac{2-p_{0}}{p_{0}}} t\right)$ and $\partial_{t} \tilde{v}(x, t)=\lambda^{\frac{1-p_{0}}{p_{0}}} v_{t}\left(x, \lambda^{\frac{2-p_{0}}{p_{0}}} t\right)$ for all $(x, t) \in Q_{2 \varrho}$. Moreover, we observe the rescaled vector-field $\tilde{a}(\cdot)$ and the rescaled inhomogeneities $\tilde{F}$ and $\tilde{f}$ as follows:

$$
\tilde{a}(x, t, w):=\lambda^{\frac{1-p_{0}}{p_{0}}} a\left(x, \lambda^{\frac{2-p_{0}}{p_{0}}} t, \lambda^{\frac{1}{p_{0}}} w\right), \quad \tilde{F}(x, t):=\lambda^{-\frac{1}{p_{0}}} F\left(x, \lambda^{\frac{2-p_{0}}{p_{0}}} t\right), \quad \text { and } \quad \tilde{f}(x, t):=\lambda^{\frac{1-p_{0}}{p_{0}}} f\left(x, \lambda^{\frac{2-p_{0}}{p_{0}}} t\right)
$$

for all $(x, t) \in Q_{2 \varrho}$ and $w \in \mathbb{R}^{n}$. Then, $\tilde{v}$ is a weak solution of the parabolic equation

$$
\partial_{t} \tilde{v}-\operatorname{div} \tilde{a}(x, t, D \tilde{v})=\tilde{f}-\operatorname{div}\left(|\tilde{F}|^{\tilde{p}(\cdot)-2} \tilde{F}\right) \quad \text { in } Q_{2 \varrho},
$$


i.e. for the rescaled parabolic problem of the parabolic equation (1). The next step is to ensure that the rescaled exponent $\tilde{p}$ and the rescaled vector-field $\tilde{a}(\cdot)$ satisfy the conditions (6) and also the structure condition, i.e. the growth and monotonicity property (2) and (3). Since, $p_{0} \geq 2, \lambda \geq 1$ and therefore $\lambda^{\frac{2-p_{0}}{2 p_{0}}} \leq 1$, we have

$$
\begin{aligned}
\left|\tilde{p}\left(x_{1}, t_{1}\right)-\tilde{p}\left(x_{2}, t_{2}\right)\right| & \left.=\left|p\left(x_{1}, \lambda^{\frac{2-p_{0}}{p_{0}}} t_{1}\right)-p\left(x_{2}, \lambda^{\frac{2-p_{0}}{p_{0}}} t_{2}\right)\right| \leq \omega\left(\max \left\{\left|x_{1}-x_{2}\right|, \sqrt{\lambda^{\frac{2-p_{0}}{p_{0}}}\left|t_{1}-t_{2}\right|}\right)\right\}\right) \\
& =\omega\left(\max \left\{\left|x_{1}-x_{2}\right|, \lambda^{\frac{2-p_{0}}{p_{0}}} \sqrt{\left|t_{1}-t_{2}\right|}\right\}\right) \leq \omega\left(\max \left\{\left|x_{1}-x_{2}\right|, \sqrt{\left|t_{1}-t_{2}\right|}\right\}\right)=\omega\left(d_{\mathcal{P}}\left(z_{1}, z_{2}\right)\right),
\end{aligned}
$$

where we used (6). Thus, we have shown

$$
\left|\tilde{p}\left(x_{1}, t_{1}\right)-\tilde{p}\left(x_{2}, t_{2}\right)\right| \leq \omega\left(d_{\mathcal{P}}\left(\left(x_{1}, t_{1}\right),\left(x_{2}, t_{2}\right)\right)\right)
$$

for any choice of $\left(x_{1}, t_{1}\right),\left(x_{2}, t_{2}\right) \in Q_{2 \varrho}$. Next, we check the assumptions (2) and (3) for the vector-field $\tilde{a}$. Therefore, we apply (2) to the vector-field $\tilde{a}(\cdot)$. This yields

$$
\begin{aligned}
|\tilde{a}(x, t, w)| & =\left|\lambda^{\frac{1-p_{0}}{p_{0}}} a\left(x, \lambda^{\frac{2-p_{0}}{p_{0}}} t, \lambda^{\frac{1}{p_{0}}} w\right)\right|=\lambda^{\frac{1-p_{0}}{p_{0}}}\left|a\left(x, \lambda^{\frac{2-p_{0}}{p_{0}}} t, \lambda^{\frac{1}{p_{0}}} w\right)\right| \leq \lambda^{\frac{1-p_{0}}{p_{0}}} L\left(1+\left|\lambda^{\frac{1}{p_{0}}} w\right|\right)^{p\left(x, \lambda^{\frac{2-p_{0}}{p_{0}}} t\right)-1} \\
& =\lambda^{\frac{1-p_{0}}{p_{0}}} L\left(1+\lambda^{\frac{1}{p_{0}}}|w|\right)^{\tilde{p}(\cdot)-1} \leq \lambda^{\frac{\tilde{p}(\cdot)-p_{0}}{p_{0}}} L(1+|w|)^{\tilde{p}(\cdot)-1} \leq \lambda^{p_{2}-p_{1}} L(1+|w|)^{\tilde{p}(\cdot)-1} \leq \hat{c} L(1+|w|)^{\tilde{p}(\cdot)-1}
\end{aligned}
$$

for all $(x, t) \in Q_{2 \varrho}$ and $w \in \mathbb{R}^{n}$, where we finally used (20). Thus, we have

$$
|\tilde{a}(x, t, w)| \leq \hat{c} L(1+|w|)^{\tilde{p}(\cdot)-1}
$$

for all $(x, t) \in Q_{2 \varrho}$ and $w \in \mathbb{R}^{n}$. Finally, we can conclude that

$$
\begin{aligned}
\left(\tilde{a}(x, t, w)-\tilde{a}\left(x, t, w_{0}\right)\right) \cdot\left(w-w_{0}\right) & =\lambda^{-\frac{p_{0}}{p_{0}}}\left(a\left(x, \lambda^{\frac{2-p_{0}}{p_{0}}} t, \lambda^{\frac{1}{p_{0}}} w\right)-a\left(x, \lambda^{\frac{2-p_{0}}{p_{0}}} t, \lambda^{\frac{1}{p_{0}}} w_{0}\right)\right) \cdot\left(\lambda^{\frac{1}{p_{0}}} w-\lambda^{\frac{1}{p_{0}}} w_{0}\right) \\
& \geq \lambda^{-\frac{p_{0}}{p_{0}}} \mu\left(s^{2}+\left|\lambda^{\frac{1}{p_{0}}} w\right|^{2}+\left|\lambda^{\frac{1}{p_{0}}} w_{0}\right|^{2}\right)^{\frac{\tilde{p}(-)-2}{2}}\left|\lambda^{\frac{1}{p_{0}}} w-\lambda^{\frac{1}{p_{0}}} w_{0}\right|^{2} \\
& =\lambda^{\frac{\tilde{p}(-)-p_{0}}{p_{0}}} \mu\left(\lambda^{-\frac{2}{p_{0}}} s^{2}+|w|^{2}+\left|w_{0}\right|^{2}\right)^{\frac{\tilde{p}(-)-2}{2}}\left|w-w_{0}\right|^{2} \\
& \geq \frac{\mu}{\hat{c}}\left(\lambda^{-\frac{2}{p_{0}}} s^{2}+|w|^{2}+\left|w_{0}\right|^{2}\right)^{\frac{\tilde{p}(-)-2}{2}}\left|w-w_{0}\right|^{2}
\end{aligned}
$$

for all $(x, t) \in Q_{2 \varrho}$ and $w, w_{0} \in \mathbb{R}^{n}$, since $\lambda^{\frac{p_{(}(-)-p_{0}}{p_{0}}}=\lambda^{-\frac{p_{0}-\bar{p}()}{p_{0}}} \geq \lambda^{-\left(p_{2}-p_{1}\right)} \geq \hat{c}^{-1}$, where we used (20) for the last step. This yields the desired assumption

$$
\left(\tilde{a}(x, t, w)-\tilde{a}\left(x, t, w_{0}\right)\right) \cdot\left(w-w_{0}\right) \geq \mu\left(\tilde{s}^{2}+|w|^{2}+\left|w_{0}\right|^{2}\right)^{\frac{\tilde{p}(\cdot)-2}{2}}\left|w-w_{0}\right|^{2}
$$

for all $(x, t) \in Q_{2 \varrho}$ and $w, w_{0} \in \mathbb{R}^{n}$ with $s$ replaced by $\tilde{s}:=s \lambda^{-\frac{1}{p_{0}}} \in(0,1)$. Now, we are in the situation to apply Theorem 6 with $\left(\frac{\mu}{\hat{c}}, \hat{c} L\right)$ instead of $(\mu, L)$. Therefore, we conclude that $D \tilde{v} \in L_{\text {loc }}^{p(\cdot)\left(1+\varepsilon_{0}\right)}\left(Q_{2 \varrho}, \mathbb{R}^{n}\right)$ and moreover the following quantitative estimate holds:

$$
f_{Q_{\varrho}}|D \tilde{v}|^{p(\cdot)\left(1+\varepsilon_{0}\right)} \mathrm{d} z \leq c\left(f_{Q_{2 \varrho}}\left(|D \tilde{v}|^{\tilde{p}(\cdot)}+|\tilde{F}|^{\tilde{p}(\cdot)}+|\tilde{f}|^{\gamma_{1}^{\prime}}+1\right) \mathrm{d} z\right)^{1+\varepsilon_{0} d}+c f_{Q_{2 \varrho}}\left(|\tilde{F}|^{\tilde{p}(\cdot)}+|\tilde{f}|^{\gamma_{1}^{\prime}}+1\right)^{\left(1+\varepsilon_{0}\right)} \mathrm{d} z
$$

for a constant $c=c\left(n, \gamma_{1}, \gamma_{2}, \mu, L, \hat{c}\right)$. Notice that $p_{0}=p(0)=\tilde{p}(0)$. Next, we transform from $v$ to $\tilde{v}$, use the 
previous estimate and then, we scale back from $\tilde{v}$ to $v$. This yields, using (20), (12) and (21), that

$$
\begin{aligned}
& f_{Q_{\varrho}^{(\lambda)}}|D \nu|^{p(\cdot)\left(1+\varepsilon_{0}\right)}=\int_{Q_{\varrho}} \lambda^{\frac{\tilde{p}(\cdot)}{p_{0}}\left(1+\varepsilon_{0}\right)}|D \tilde{v}|^{\tilde{p}(\cdot)\left(1+\varepsilon_{0}\right)} \mathrm{d} z=\int_{Q_{\varrho}} \lambda^{\frac{\tilde{p}(\cdot)-p_{1}+p_{1}}{p_{0}}\left(1+\varepsilon_{0}\right)}|D \tilde{v}|^{\tilde{p}(\cdot)\left(1+\varepsilon_{0}\right)} \mathrm{d} z \\
& \leq \int_{Q_{\varrho}} \lambda^{\frac{p_{2}-p_{1}}{p_{0}}\left(1+\varepsilon_{0}\right)} \lambda^{\frac{p_{1}}{p_{0}}\left(1+\varepsilon_{0}\right)}|D \tilde{v}|^{\tilde{p}(\cdot)\left(1+\varepsilon_{0}\right)} \mathrm{d} z \leq c \lambda^{1+\varepsilon_{0}} \int_{Q_{\varrho}}|D \tilde{v}|^{\tilde{p}(\cdot)\left(1+\varepsilon_{0}\right)} \mathrm{d} z \\
& \leq c \lambda^{1+\varepsilon_{0}}\left(f_{Q_{2 \varrho}}\left(|D \tilde{v}|^{\tilde{p}(\cdot)}+|\tilde{F}|^{\tilde{p}(\cdot)}+|\tilde{f}|^{\gamma_{1}^{\prime}}+1\right) \mathrm{d} z\right)^{1+\varepsilon_{0} d}+c \lambda^{1+\varepsilon_{0}} \int_{Q_{2 \varrho}}\left(|\tilde{F}|^{\tilde{p}(\cdot)}+|\tilde{f}|^{\gamma_{1}^{\prime}}+1\right)^{\left(1+\varepsilon_{0}\right)} \mathrm{d} z \\
& \leq c \lambda^{\varepsilon_{0}(1-d)}\left(f_{Q_{2 \varrho}^{(\lambda)}}|D \nu|^{p(\cdot)} \mathrm{d} z\right)^{1+\varepsilon_{0} d}+c \lambda^{1+\varepsilon_{0}} \int_{Q_{2 \varrho}^{(\lambda)}}\left(|F|^{p(\cdot)}+|f|^{\gamma_{1}^{\prime}}\right)^{\left(1+\varepsilon_{0}\right)} \mathrm{d} z+c \lambda^{1+\varepsilon_{0}} \\
& =c \lambda^{\varepsilon_{0}(1-d)}\left(f_{Q_{2 \varrho}^{(\lambda)}}|D v|^{p(\cdot)} \mathrm{d} z\right)^{1+\varepsilon_{0} d}+c \lambda^{1+\varepsilon_{0}} \int_{Q_{2 \varrho}^{(\lambda)}}\left(|F|^{p(\cdot)}+|f|^{\gamma_{1}^{\prime}}\right)^{\left(1+\varepsilon_{0}\right)} \mathrm{d} z+c \lambda^{1+\varepsilon_{0}} \\
& \leq c \lambda^{\varepsilon_{0}(1-d)}\left(c_{*} \lambda\right)^{1+\varepsilon_{0} d}+c \cdot\left(c_{*} \lambda\right)^{1+\varepsilon_{0}}+c \lambda^{1+\varepsilon_{0}}=c \lambda^{1+\varepsilon_{0}} \text {. }
\end{aligned}
$$

with a constant $c=c\left(n, \gamma_{1}, \gamma_{2}, \mu, L, K, c_{*}, \hat{c}\right)$. This finishes the proof of the lemma in the case $p_{0} \geq 2$.

Finally, we have to prove the case $\frac{2 n}{n+2}<p_{0}<2$. Here, we define $\tilde{p}, \tilde{v}, \tilde{F}, \tilde{f}$ and $\tilde{a}$ similarly as above, i.e. $\tilde{p}(x, t):=p\left(\lambda^{\frac{p_{0}-2}{2 p_{0}}} x, t\right), \tilde{v}(x, t):=\lambda^{-\frac{1}{2}} v\left(\lambda^{\frac{p_{0}-2}{2 p_{0}}} x, t\right), \tilde{F}(x, t):=\lambda^{-\frac{1}{p_{0}}} F\left(\lambda^{\frac{p_{0}-2}{2 p_{0}}} x, t\right), \tilde{f}(x, t):=\lambda^{-\frac{1}{2}} f\left(\lambda^{\frac{p_{0}-2}{2 p_{0}}} x, t\right)$, and

$$
\tilde{a}(x, t, w):=\lambda^{\frac{1-p_{0}}{p_{0}}} a\left(\lambda^{\frac{p_{0}-2}{2 p_{0}}} x, t, \lambda^{-\frac{1}{p_{0}}} w\right)
$$

for $(x, t) \in Q_{2 \varrho}$, where $\tilde{\varrho}:=\lambda^{\frac{p_{0}-2}{2 p_{0}}} \varrho$. A straightforward computation shows that $\tilde{v}$ is a weak solution of the equation

$$
\partial_{t} \tilde{v}-\operatorname{div} \tilde{a}(x, t, D \tilde{v})=\tilde{f}-\operatorname{div}\left(|\tilde{F}|^{\tilde{p}(\cdot)-2} \tilde{F}\right) \text { in } Q_{2 \tilde{\varrho}},
$$

where $\tilde{a}, \tilde{v}, \tilde{p}, \tilde{F}$ and $\tilde{f}$ are the time quantities defined just above. Notice, that $\tilde{a}$ satisfies the growth and monotonicity condition (24) and (25). Similar to (23), we can also conclude that $\left|\tilde{p}\left(z_{1}\right)-\tilde{p}\left(z_{2}\right)\right| \leq \omega\left(d_{\mathcal{P}}\left(z_{1}, z_{2}\right)\right)$ is valid, for every $z_{1}, z_{2} \in Q_{2}$, since $p_{0}<2$ and $\lambda \geq 1$. Applying again Theorem 6 and repeating the computations from above we obtain the assertion of the lemma also in the case $p_{0}<2$.

A priori estimates. For the proof of the Calderón-Zygmund estimates for the spatial gradient we will need a gradient estimate. To this aim, we refer in the next theorem Lipschitz bounds for solutions to parabolic equations with standard growth, that will be employed for suitable comparison problems. These Lipschitz bounds will be very important for the proof of the gradient estimate via comparison and they are due to the fundamental contributions of DiBenedetto and Friedman (DiBenedetto \& Friedman, 1985a,b) and can be retrieved from (DiBenedetto, 1993, Chapter 8). Later on, we will transfer these a priori estimates via comparison argument to our nonstandard growth problem and mainly, to our parabolic obstacle problem. Therefore, we denote

$$
C_{\varrho}^{(\lambda)}\left(z_{0}\right):=B_{\varrho}\left(x_{0}\right) \times\left(t_{0}-\lambda^{\frac{2-p}{p}} \varrho^{2}, t_{0}+\lambda^{\frac{2-p}{p}} \varrho^{2}\right)
$$

Note that the scaling of cylinders $C_{\varrho}^{(\lambda)}\left(z_{0}\right)$ does not depends on the center $z_{0}$. Later on, we will apply the subsequent Theorem with the choice $p=p_{0} \equiv p\left(z_{0}\right)$. Thus, the cylinder $C_{\varrho}^{(\lambda)}\left(z_{0}\right)$ becomes the intrinsic cylinder $Q_{\varrho}^{(\lambda)}\left(z_{0}\right)$. The precise statement of this result reads as follows and was established in (Scheven, 2014, Theorem 5.3).

Theorem 9. Suppose that the vector-field $b: \Omega_{T} \times \mathbb{R}^{n} \rightarrow \mathbb{R}^{n}$ satisfies (2)-(5) with $p(\cdot)=p=$ const. and a( $\left.\cdot\right)$ replaced by $b(\cdot)$ and furthermore, that $b(\cdot)$ is differentiable with respect to the spatial variable with

$$
\left|D_{x} b(z, w)\right| \leq \gamma L(1+|w|)^{p-1}
$$

for all $z \in \Omega_{T}$ and $w \in \mathbb{R}^{n}$, where $\gamma \geq 0$ is a fixed constant. Assume that $w \in C^{0}\left([0, T] ; L^{2}(\Omega)\right) \cap L^{p}\left(0, T ; W^{1, p}(\Omega)\right)$ is a weak solution of the parabolic equation $\partial_{t} w-\operatorname{div} b(z, D w)=0$ on $\Omega_{T}$ and that $C_{2 \varrho}^{(\lambda)}\left(z_{0}\right) \subset \Omega_{T}$ is an intrinsic cylinder with

$$
\int_{C_{2 \varrho}^{(\lambda)}\left(z_{0}\right)}|D w|^{p} \mathrm{~d} z \leq c_{*} \lambda .
$$


Then, there holds

$$
\sup _{C_{\alpha}^{(\lambda)}\left(z_{0}\right)}|D w| \leq c_{\text {Lip }} \lambda^{\frac{1}{p}}
$$

where the constant $c_{\text {Lip }}$ depends only on $p, n, \mu, L, \gamma$ and $c_{*}$.

In section 2.3 we will prove the gradient estimate via comparison. For this aim, we will apply the following comparison lemma which reads as follows:

Lemma 10. Assume that the vector-fields $a, b: Q_{\varrho}^{(\lambda)}\left(z_{0}\right) \times \mathbb{R}^{n} \rightarrow \mathbb{R}^{n}$ satisfy the growth condition (2) and $b(\cdot)$ additionally the monotonicity property (3) with exponents $p(\cdot)$, which fulfil (6)-(7). Further, let $F \in L^{p(\cdot)}\left(Q_{\varrho}^{(\lambda)}\left(z_{0}\right), \mathbb{R}^{n}\right)$, $f \in L^{\gamma_{1}^{\prime}}\left(Q_{\varrho}^{(\lambda)}\left(z_{0}\right), \mathbb{R}\right)$ and assume that $v, w \in C^{0}\left(\Lambda_{\varrho}^{(\lambda)}\left(t_{0}\right) ; L^{2}\left(B_{\varrho}\left(x_{0}\right)\right)\right) \cap W^{p(\cdot)}\left(Q_{\varrho}^{(\lambda)}\left(z_{0}\right)\right)$ are solutions of the parabolic equations

$$
\partial_{t} v-\operatorname{div} a(z, D v)=f-\operatorname{div}\left(|F|^{p(\cdot)-2} F\right) \text { in } Q_{\varrho}^{(\lambda)}\left(z_{0}\right)
$$

respectively

$$
\partial_{t} w-\operatorname{div} b(z, D w)=0 \quad \text { in } Q_{\varrho}^{(\lambda)}\left(z_{0}\right)
$$

with $v=w$ on $\partial_{\mathcal{P}} Q_{\varrho}^{(\lambda)}\left(z_{0}\right)$. Then, we have a comparison estimate of the form

$$
\begin{aligned}
\int_{Q_{\varrho}^{(\lambda)}\left(z_{0}\right)}|D v-D w|^{p(\cdot)} \mathrm{d} z & \leq \tilde{\kappa} \int_{Q_{\varrho}^{(\lambda)}\left(z_{0}\right)}(s+|D v|)^{p(\cdot)} \mathrm{d} z+c_{\tilde{\kappa}} \int_{Q_{\varrho}^{(\lambda)}\left(z_{0}\right)}|a(z, D v)-b(z, D v)|^{p^{\prime}(\cdot)} \mathrm{d} z \\
& +c_{\tilde{\kappa}} \int_{Q_{\varrho}^{(\lambda)}\left(z_{0}\right)}|F|^{p(\cdot)}+|f|^{\gamma_{1}^{\prime}}+1 \mathrm{~d} z
\end{aligned}
$$

for every radius $\varrho \in(0,1]$ and $\tilde{\kappa} \in(0,1)$ with a constant $c_{\tilde{\kappa}}=c\left(\tilde{\kappa}, n, \gamma_{1}, \gamma_{2}, \mu, L\right)$. Once again, in the case $p(\cdot) \geq 2$, we may omit the first integral on the right-hand side, and the constant does not depend on $\tilde{\kappa}$. Furthermore, for every exponent $p(\cdot)>\frac{2 n}{n+2}$, there holds the energy estimate

$$
\begin{aligned}
\int_{Q_{\varrho}^{(\lambda)}\left(z_{0}\right)}|D w|^{p(\cdot)} \mathrm{d} z & \leq c\left(\gamma_{1}, \gamma_{2}\right) \int_{Q_{\varrho}^{(\lambda)}\left(z_{0}\right)}(s+|D v|)^{p(\cdot)} \mathrm{d} z+c \int_{Q_{\varrho}^{(\lambda)}\left(z_{0}\right)}|a(z, D v)-b(z, D v)|^{p^{(}(\cdot)} \mathrm{d} z \\
& +c \int_{Q_{\varrho}^{(\lambda)}\left(z_{0}\right)}|F|^{p(\cdot)}+|f|^{\gamma_{1}^{\prime}}+1 \mathrm{~d} z
\end{aligned}
$$

where $c=c\left(n, \gamma_{1}, \gamma_{2}, \mu, L\right)$ and $\varrho \in(0,1]$.

Proof. First, we consider the weak formulation of the parabolic equations (27) and (28) and test the equations with $v-w \in C^{0}\left(\Lambda_{\varrho}^{(\lambda)}\left(t_{0}\right) ; L^{2}\left(B_{\varrho}\left(x_{0}\right)\right)\right) \cap W_{0}^{p(\cdot)}\left(Q_{\varrho}^{(\lambda)}\left(z_{0}\right)\right)$. Subtracting the resulting equations, we get

$$
\begin{gathered}
\left\langle\left\langle\partial_{t} v-\partial_{t} w, v-w\right\rangle\right\rangle_{Q_{\varrho}^{(\lambda)}\left(z_{0}\right)}+\int_{Q_{\varrho}^{(\lambda)}\left(z_{0}\right)}(a(z, D v)-b(z, D w)) \cdot D(v-w) \mathrm{d} z \\
=\int_{Q_{\varrho}^{(\lambda)}\left(z_{0}\right)} f(v-w) \mathrm{d} z+\int_{Q_{\varrho}^{(\lambda)}\left(z_{0}\right)}\left(|F|^{p(\cdot)-2} F\right) \cdot D(v-w) \mathrm{d} z .
\end{gathered}
$$

Moreover, we can conclude that

$$
\begin{aligned}
\left\langle\left\langle\partial_{t} v-\right.\right. & \left.\left.\partial_{t} w, v-w\right\rangle\right\rangle_{Q_{\varrho}^{(\lambda)}\left(z_{0}\right)}+\int_{Q_{\varrho}^{(\lambda)}\left(z_{0}\right)}(b(z, D v)-b(z, D w)) \cdot D(v-w) \mathrm{d} z \\
& =\int_{Q_{\varrho}^{(\lambda)}\left(z_{0}\right)} f(v-w) \mathrm{d} z+\int_{Q_{\varrho}^{(\lambda)}\left(z_{0}\right)}\left(|F|^{p(\cdot)-2} F\right) \cdot D(v-w) \mathrm{d} z+\int_{Q_{\varrho}^{(\lambda)}\left(z_{0}\right)}(b(z, D v)-a(z, D v)) \cdot D(v-w) \mathrm{d} z \\
& \leq c_{\varepsilon}\left(\int_{Q_{\varrho}^{(\lambda)}\left(z_{0}\right)}|F|^{p(\cdot)}+|f|^{\gamma_{1}^{\prime}}+1 \mathrm{~d} z\right)+c_{\varepsilon}\left(\gamma_{1}, \gamma_{2}, \varepsilon\right) \int_{Q_{\varrho}^{(\lambda)}\left(z_{0}\right)}|a(z, D v)-b(z, D v)|^{p^{(}(\cdot)} \mathrm{d} z \\
& +c\left(\gamma_{1}, \gamma_{2}\right) \varepsilon \int_{Q_{\varrho}^{(\lambda)}\left(z_{0}\right)}|D(v-w)|^{p(\cdot)}+|v-w|^{\gamma_{1}} \mathrm{~d} z
\end{aligned}
$$


for $\varrho \in(0,1]$ with a constant $c=c\left(\gamma_{1}, \gamma_{2}, L\right)$ and $\varepsilon \in(0,1)$, where we used Young's inequality. Next, we treat the first integral on the left-hand side as usual to infer that this integral is non-negative. Therefore, we use Lemma 2 and the fact that $u(\cdot, 0)=v(\cdot, 0)$. This yields

$$
2\left\langle\left\langle\partial_{t} v-\partial_{t} w, v-w\right\rangle\right\rangle_{Q_{Q}^{(\lambda)}\left(z_{0}\right)}=\int_{B_{Q}\left(x_{0}\right)}|v(x, t)-w(x, t)|^{2} d x \geq 0
$$

for all $t \in \Lambda_{\varrho}^{(\lambda)}\left(t_{0}\right)$. Further, we apply the monotonicity condition (3) to (31). This yields

$$
\begin{aligned}
\int_{Q_{Q}^{(\lambda)}\left(z_{0}\right)}\left(s^{2}+|D v|^{2}+|D w|^{2}\right)^{\frac{p(\cdot)-2}{2}}|D v-D w|^{2} \mathrm{~d} z & \leq c\left(\int_{Q_{\varrho}^{(\lambda)}\left(z_{0}\right)}|F|^{p(\cdot)}+|f|^{\gamma_{1}^{\prime}}+1 \mathrm{~d} z\right) \\
& +c_{\varepsilon}\left(\gamma_{1}, \gamma_{2}, \varepsilon\right) \int_{Q_{\varrho}^{(\lambda)}\left(z_{0}\right)}|a(z, D v)-b(z, D v)|^{p^{\prime}(\cdot)} \mathrm{d} z \\
& +c\left(\gamma_{1}, \gamma_{2}\right) \varepsilon \int_{Q_{\varrho}^{(\lambda)}\left(z_{0}\right)}|D(v-w)|^{p(\cdot)}+1 \mathrm{~d} z
\end{aligned}
$$

for $\varrho \in(0,1]$ with a constant $c=c\left(\gamma_{1}, \gamma_{2}, L\right)$ and $\varepsilon \in(0,1)$. In the case $p(\cdot) \geq 2$, this implies immediately the comparison estimate (29) by choosing $\varepsilon=\frac{1}{2 c\left(\gamma_{1}, \gamma_{2}\right)}$. Therefore, we have only to prove the case $\frac{2 n}{n+2}<p(\cdot)<2$. For this aim, we use Young's inequality with exponents $\frac{2}{2-p(\cdot)}$ and $\frac{2}{p(\cdot)}$ in order to estimate, for any $\delta>0$

$$
\begin{aligned}
|D v-D w|^{p(\cdot)} & \leq \delta\left(s^{2}+|D v|^{2}+|D w|^{2}\right)^{\frac{p(\cdot)}{2}}+\delta^{1-\frac{2}{p(\cdot)}}\left(s^{2}+|D v|^{2}+|D w|^{2}\right)^{\frac{p(\cdot)-2}{2}}|D v-D w|^{2} \\
& \leq \delta\left(s^{2}+|D v|^{2}+|D w|^{2}\right)^{\frac{p(\cdot)-2}{2}}\left(s^{2}+3|D v|^{2}+2|D w-D v|^{2}\right)+\delta^{1-\frac{2}{p(\cdot)}}\left(s^{2}+|D v|^{2}+|D w|^{2}\right)^{\frac{p(\cdot)-2}{2}}|D v-D w|^{2} \\
& \leq 8 \delta(s+|D v|)^{p(\cdot)}+c\left(\gamma_{1}, \gamma_{2}, \delta\right)\left(s^{2}+|D v|^{2}+|D w|^{2}\right)^{\frac{p(\cdot-2}{2}}|D v-D w|^{2},
\end{aligned}
$$

where we used the fact $p(\cdot)-2<0$ for the last estimate. Integrating this estimate over $Q_{\varrho}^{(\lambda)}\left(z_{0}\right)$, estimating the right-hand side further by (32) and finally, choosing $\varepsilon=\frac{1}{2 c\left(\gamma_{1}, \gamma_{2}\right)}$ and $\delta=\frac{\tilde{\kappa}}{16}$. This implies the claimed comparison estimate (29). The second claim (30) is again an easy consequence of (29).

\subsection{Proof of the Comparison Estimate}

First, we let $K \geq 1$ and suppose that (12) is valid. Next, we fix $\kappa, H \geq 1$ to be specified later. In the following, we consider a cylinder $Q_{\varrho}^{(\lambda)}\left(z_{0}\right)=B_{\varrho}\left(x_{0}\right) \times \Lambda_{\varrho}^{(\lambda)}\left(t_{0}\right)$ with center $z_{0}=\left(x_{0}, t_{0}\right) \in \Omega_{T}, \varrho \in(0,1]$, where $\lambda \geq 1$ and which satisfies $Q_{2 \varrho}^{(\lambda)}\left(z_{0}\right) \Subset \Omega_{T}$ and

$$
\frac{\lambda}{\kappa} \leq f_{Q_{2 \varrho}^{(\lambda)}\left(z_{0}\right)}|D u|^{p(\cdot)} \mathrm{d} z+f_{Q_{2 \varrho}^{(\lambda)}\left(z_{0}\right)} H\left(|F|^{p(\cdot)}+|f|^{\gamma_{1}^{\prime}}+1\right) \mathrm{d} z \leq \lambda .
$$

Then, we denote by $v \in W\left(Q_{2 \varrho}^{(\lambda)}\left(z_{0}\right)\right)$ the unique solution of the initial-boundary value problems

$$
\left\{\begin{aligned}
\partial_{t} v-\operatorname{div} a(z, D v) & =0 & & \text { in } Q_{2 \varrho}^{(\lambda)}\left(z_{0}\right), \\
v & =u & & \text { on } \partial_{\mathcal{P}} Q_{2 \varrho}^{(\lambda)}\left(z_{0}\right) .
\end{aligned}\right.
$$

Moreover, the function $w \in C^{0}\left(\Lambda_{\varrho}^{(\lambda)}\left(t_{0}\right) ; L^{2}\left(B_{\varrho}\left(x_{0}\right)\right)\right) \cap L^{p_{0}}\left(\Lambda_{\varrho}^{(\lambda)}\left(t_{0}\right) ; W^{1, p_{0}}\left(B_{\varrho}\left(x_{0}\right)\right)\right)$ denotes the unique solution of the homogeneous initial-boundary value problem

$$
\left\{\begin{aligned}
\partial_{t} w-\operatorname{div} a\left(z_{0}, D w\right) & =0 & & \text { in } Q_{\varrho}^{(\lambda)}\left(z_{0}\right), \\
w & =v & & \text { on } \partial_{\mathcal{P}} Q_{\varrho}^{(\lambda)}\left(z_{0}\right),
\end{aligned}\right.
$$

where $a(\cdot)$ denotes the vector-field which satisfies the structure assumptions (2)-(9). Notice that the existence of $v$ and $w$ is satisfied by (Erhardt, 2013a).

Step 1: Proof of the first comparison estimate. We start by comparing the parabolic problem (1) with the parabolic boundary problem (34). Here, we apply Lemma 10 to conclude the first comparison estimate

$$
\begin{aligned}
\int_{Q_{2 \varrho}^{(\lambda)}\left(z_{0}\right)}|D u-D v|^{p(\cdot)} \mathrm{d} z & \leq \tilde{\kappa} \int_{Q_{2 \varrho}^{(\lambda)}\left(z_{0}\right)}(s+|D u|)^{p(\cdot)} \mathrm{d} z+c_{\tilde{\kappa}} \int_{Q_{2 \varrho}^{(\lambda)}\left(z_{0}\right)}|D u|^{p(\cdot)}+|F|^{p(\cdot)}+|f|^{\gamma_{1}^{\prime}}+1 \mathrm{~d} z \\
& \leq c\left(\tilde{\kappa}+\frac{c_{\tilde{\kappa}}}{H}\right) \lambda\left|Q_{2 \varrho}^{(\lambda)}\left(z_{0}\right)\right|
\end{aligned}
$$


for every $\tilde{\kappa} \in(0,1)$ and $\varrho \in(0,1]$ with constants $c_{\tilde{\kappa}}=c\left(\tilde{\kappa}, n, \gamma_{1}, \gamma_{2}, \mu, L\right)$ and $c=c\left(n, \gamma_{1}, \gamma_{2}, \mu, L\right)$, where we used (33). Furthermore, we have from (30) the following energy estimate

$$
\int_{Q_{2 \varrho}^{(\lambda)}\left(z_{0}\right)}|D v|^{p(\cdot)} \mathrm{d} z \leq c \lambda\left|Q_{2 \varrho}^{(\lambda)}\left(z_{0}\right)\right|
$$

for every $\varrho \in(0,1]$ with a constant $c=c\left(n, \gamma_{1}, \gamma_{2}, \mu, L\right)$, where we also used (33).

Step 2: Proof of the higher integrability. Now, we let $\varepsilon_{0}=\varepsilon_{0}\left(n, \gamma_{1}, \gamma_{2}, \mu, L, \sigma\right)>0$ be the higher integrability exponent from Corollary 8 and set

$$
\varrho_{3}:=\min \left\{\varrho_{1}, \frac{\varrho_{2}}{2}, 1\right\} \in(0,1]
$$

where $\varrho_{2}$ is the radius from Lemma 7 and $\varrho_{1}$ the one for the higher integrability from Corollary 8 with the choices $f, F=0$. Therefore, $\varrho_{3}$ depends on $n, \gamma_{1}, \gamma_{2}, \mu, L, \kappa, K, H, \omega(\cdot)$. In the course of the proof, we shall further reduce the value of $\varrho_{3}$ when necessary, but without changing its dependencies. In the following, we assume that

$$
\varrho \leq \varrho_{3} .
$$

Due to assumption (33) we are allowed to apply Lemma 7 on $Q_{2 \varrho}^{(\lambda)}\left(z_{0}\right)$ which yields that

$$
p_{2}-p_{1} \leq \omega\left(\tilde{\Gamma}(2 \varrho)^{\alpha}\right) \text { and } \lambda^{p_{2}-p_{1}} \leq \lambda^{\omega\left(\tilde{\Gamma}(2 \varrho)^{\alpha}\right)} \leq e^{\frac{3 n L_{1} p_{0}}{\alpha}} \leq e^{\frac{3 n L_{1} \gamma_{2}}{\alpha}},
$$

since $\lambda \geq 1$, where $\tilde{\Gamma}$ and $\alpha$ are defined in (19). Here, we set $\hat{c}=\hat{c}\left(n, \gamma_{1}, \gamma_{2}, L_{1}\right):=e^{\frac{3 n L_{1} \gamma_{2}}{\alpha}}$. Thus, assumption (20) from Corollary 8 is valid. The assumption (21) is also fulfilled by the energy estimate (37), where have to replace the constant $c$ by the constant $\hat{c}$. The application of the Corollary 8 ensures that $D v \in L^{p(\cdot)\left(1+\varepsilon_{0}\right)}\left(Q_{2 \varrho}^{(\lambda)}, \mathbb{R}^{n}\right)$ and moreover, the following higher integrability estimate

$$
\int_{Q_{\varrho}^{(\lambda)}\left(z_{0}\right)}|D \nu|^{p(\cdot)\left(1+\varepsilon_{0}\right)} \mathrm{d} z \leq c \lambda^{1+\varepsilon_{0}} .
$$

Notice, since we have from (38) that $\varrho \leq \varrho_{1}$, we are allowed to apply Corollary 8 . Next, we reduce the value of $\varrho_{3}$, such that

$$
\omega\left(\tilde{\Gamma}\left(2 \varrho_{3}\right)^{\alpha}\right) \leq \frac{\varepsilon_{1}}{\gamma_{1}^{\prime}}, \quad \text { where } \varepsilon_{1}:=\sqrt{1+\varepsilon_{0}}-1 \leq \varepsilon_{0}
$$

is satisfied. Thus, by (39), for any $z \in Q_{2 \varrho}^{(\lambda)}\left(z_{0}\right)$ there holds

$$
p_{0}\left(1+\varepsilon_{1}\right) \leq p(\cdot)\left(1+\omega\left(\tilde{\Gamma}(2 \varrho)^{\alpha}\right)\right)\left(1+\varepsilon_{1}\right) \leq p(\cdot)\left(1+\omega\left(\tilde{\Gamma}\left(2 \varrho_{3}\right)^{\alpha}\right)\right)\left(1+\varepsilon_{1}\right)<p(\cdot)\left(1+\varepsilon_{1}\right)^{2}=p(\cdot)\left(1+\varepsilon_{0}\right)
$$

Furthermore, we have $D v \in L^{p_{0}\left(1+\varepsilon_{1}\right)}\left(Q_{\varrho}^{(\lambda)}\left(z_{0}\right), \mathbb{R}^{n}\right)$ together with the following estimate

$$
\begin{aligned}
\int_{Q_{\varrho}^{(\lambda)}\left(z_{0}\right)}|D \nu|^{p_{0}\left(1+\varepsilon_{1}\right)} \mathrm{d} z & \leq \int_{Q_{\varrho}^{(\lambda)}\left(z_{0}\right)}|D \nu|^{p(\cdot)\left(1+\omega\left(\tilde{\Gamma}(2 \varrho)^{\alpha}\right)\right)\left(1+\varepsilon_{1}\right)} \mathrm{d} z+1 \leq\left(f_{Q_{\varrho}^{(\lambda)}\left(z_{0}\right)}|D \nu|^{p(\cdot)\left(1+\varepsilon_{0}\right)} \mathrm{d} z\right)^{\frac{\left(1+\omega\left(\tilde{\Gamma}(2 \varrho)^{\alpha}\right)\left(1+\varepsilon_{1}\right)\right.}{1+\varepsilon_{0}}}+1 \\
& \leq c \lambda^{\left(1+\omega\left(\tilde{\Gamma}(2 \varrho)^{\alpha}\right)\right)\left(1+\varepsilon_{1}\right)}+1=c \lambda^{\left(1+\varepsilon_{1}\right)} \lambda^{\left(1+\omega\left(\tilde{\Gamma}(2 \varrho)^{\alpha}\right)\right)}+1 \leq c \lambda^{\left(1+\varepsilon_{1}\right)}
\end{aligned}
$$

with a constant $c=c\left(n, \gamma_{1}, \gamma_{2}, \mu, L, c_{*}, \hat{c}\right)$, where we used the Hölder's inequality, (39), (40) and the fact that $\lambda \geq 1$. Finally, we observe the following

$$
p_{0}^{\prime}\left(p_{2}-1\right)=p_{0}\left(1+\frac{p_{2}-p_{0}}{p_{0}-1}\right) \leq p_{0}\left(1+\frac{\omega\left(\tilde{\Gamma}\left(2 \varrho_{3}\right)^{\alpha}\right)}{\gamma_{1}-1}\right) \leq p_{0}\left(1+\frac{\varepsilon_{1}}{\gamma_{1}}\right) \leq p_{0}\left(1+\varepsilon_{1}\right) \text {. }
$$

The previous computation combined with (42) implies

$$
\int_{Q_{\varrho}^{(\lambda)}\left(z_{0}\right)}|D v|^{p_{0}^{\prime}\left(p_{2}-1\right)} \mathrm{d} z \leq\left(\int_{Q_{\varrho}^{(\lambda)}\left(z_{0}\right)}|D v|^{p_{0}\left(1+\varepsilon_{1}\right)} \mathrm{d} z\right)^{\frac{p_{2}-1}{\left(p_{0}-1\right)\left(1+\varepsilon_{1}\right)}} \leq c \lambda^{\frac{p_{2}-1}{p_{0}-1}}=c \lambda^{1+\frac{p_{2}-p_{0}}{p_{0}-1}} \leq c \lambda
$$


with a constant $c=c\left(n, \gamma_{1}, \gamma_{2}, \mu, L, c_{*}, \hat{c}\right)$, where we used (39).

Step 3: Proof of the second comparison estimate. Next, we want to conclude a comparison estimate between $D v$ and $D w$. To compare the solution of (34) and (35), we have to consider the difference of their weak formulation (see Definition 1 with $f, F=0$ and $u$ replaced by $v$ respectively $w$ ), i.e.

$$
\int_{Q_{\varrho}^{(\lambda)}\left(z_{0}\right)}(v-w) \cdot \varphi_{t} \mathrm{~d} z-\int_{Q_{\varrho}^{(\lambda)}\left(z_{0}\right)}\left(a(z, D v)-a\left(z_{0}, D w\right)\right) \cdot D \varphi \mathrm{d} z=0
$$

for any $\varphi \in C_{0}^{\infty}\left(Q_{\varrho}^{(\lambda)}\left(z_{0}\right), \mathbb{R}^{n}\right)$. Next, we have to define for $h>0$ and $\tau:=t_{0}+\lambda^{\frac{2-p_{0}}{p_{0}}}(2 \varrho)^{2}$

$$
\chi_{h}(t):= \begin{cases}1 & \text { on }(-\infty, \tau-h], \\ -\frac{1}{h}(t-\tau) & \text { on }(\tau-h, \tau), \\ 0 & \text { on }[\tau, \infty) .\end{cases}
$$

By (42) we know that $D v \in L^{p_{0}}\left(Q_{\varrho}^{(\lambda)}\left(z_{0}\right)\right)$ with $\varrho \leq \varrho_{3}$. Thus, we have $D v-D w \in L^{p_{0}}\left(Q_{2 \varrho}^{(\lambda)}\left(z_{0}\right)\right)$ and $v=w$ on $\partial_{\mathcal{P}} Q_{\varrho}^{(\lambda)}\left(z_{0}\right)$, we are (formally) allowed to choose $\varphi=(v-w) \chi_{h}$ in the preceding identity. Then, it follows

$$
\int_{Q_{\varrho}^{(\lambda)}\left(z_{0}\right)}(v-w) \cdot \partial_{t}\left[(v-w) \chi_{h}\right] \mathrm{d} z \stackrel{h \downarrow 0}{\rightarrow}-\frac{1}{2} \int_{Q_{\varrho}^{(\lambda)}\left(z_{0}\right)}|v-w|^{2}(\cdot, \tau) d x \leq 0 .
$$

This implies

$$
\int_{Q_{\varrho}^{(\lambda)}\left(z_{0}\right)}\left(a(z, D v)-a\left(z_{0}, D w\right)\right) \cdot D(v-w) \mathrm{d} z \leq 0
$$

Moreover, we gain that

$$
\int_{Q_{\varrho}^{(\lambda)}\left(z_{0}\right)}\left(a\left(z_{0}, D v\right)-a\left(z_{0}, D w\right)\right) \cdot D(v-w) \mathrm{d} z \leq \int_{Q_{\varrho}^{(\lambda)}\left(z_{0}\right)}\left(a\left(z_{0}, D v\right)-a(z, D v)\right) \cdot D(v-w) \mathrm{d} z .
$$

Using the monotonicity property of the vector-field $a\left(z_{0}, \cdot\right)$, i.e. (3) with $p_{0}=p\left(z_{0}\right)$, we have

$$
\mu \int_{Q_{\varrho}^{(\lambda)}\left(z_{0}\right)}\left(s^{2}+|D v|^{2}+|D w|^{2}\right)^{\frac{p_{0}-2}{2}}|D(v-w)|^{2} \mathrm{~d} z \leq \int_{Q_{\varrho}^{(\lambda)}\left(z_{0}\right)}\left(a\left(z_{0}, D v\right)-a(z, D v)\right) \cdot D(v-w) \mathrm{d} z
$$

Next, we apply the continuity condition (9) and Hölder's inequality with exponents $p_{0}$ and $p_{0}^{\prime}$ to the right-hand side of the previous estimate. This yields

$$
\begin{aligned}
& \mu \int_{Q_{\varrho}^{(\lambda)}\left(z_{0}\right)}\left(s^{2}+|D v|^{2}+|D w|^{2}\right)^{\frac{p_{0}-2}{2}}|D(v-w)|^{2} \mathrm{~d} z \leq c\left(\int _ { Q _ { \varrho } ^ { ( \lambda ) } ( z _ { 0 } ) } \left(\omega\left(\lambda^{\frac{2-p_{0}}{2 p_{0}}} \varrho\right)\left[(1+|D v|)^{p_{0}-1}+(1+|D v|)^{p(\cdot)-1}\right]\right.\right. \\
& \left.\times[1+\log (1+|D v|)])^{\frac{p_{0}}{p_{0}-1}} \mathrm{~d} z\right)^{\frac{p_{0}-1}{p_{0}}}\left(\int_{Q_{\varrho}^{(\lambda)}\left(z_{0}\right)}|D(v-w)|^{p_{0}} \mathrm{~d} z\right)^{\frac{1}{p_{0}}} \\
& \leq c \omega\left(\Gamma \varrho^{\alpha}\right)\left(\int_{Q_{\varrho}^{(\lambda)}\left(z_{0}\right)}\left[(1+|D v|)^{p_{0}}+(1+|D v|)^{p_{0}^{\prime}(p(\cdot)-1)}\right]\right. \\
& \left.\times[1+\log (1+|D v|)]^{\frac{p_{0}}{p_{0}-1}} \mathrm{~d} z\right)^{\frac{p_{0}-1}{p_{0}}}\left(\int_{Q_{\varrho}^{(\lambda)}\left(z_{0}\right)}|D(v-w)|^{p_{0}} \mathrm{~d} z\right)^{\frac{1}{p_{0}}}
\end{aligned}
$$

with a constant $c=c\left(\gamma_{1}, \gamma_{2}, p_{0}, L\right)$, where we used that we have for all $z_{1}, z_{2} \in Q_{\varrho}^{(\lambda)}\left(z_{0}\right)$

$$
\omega\left(d_{\mathcal{P}}\left(z_{1}, z_{2}\right)\right) \leq \omega\left(\left|x_{1}-x_{2}\right|+\sqrt{\left|t_{1}-t_{2}\right|}\right) \leq \omega\left(2 \varrho+\sqrt{2 \lambda^{\frac{2-p_{0}}{p_{0}}} \varrho^{2}}\right) \leq \omega\left(4 \lambda^{\frac{2-\gamma_{1}}{2 p_{0}}} \varrho\right) .
$$

Lemma 7 yields $\omega(4 \varrho) \leq \omega(\Gamma \varrho)$ for $\gamma_{1} \geq 2$, since $\Gamma \geq 4$, see (18). Moreover in the case $\gamma_{1}<2$ we have

$$
\omega\left(4 \lambda^{\frac{2-\gamma_{1}}{2 p_{0}}} \varrho\right) \leq \omega\left(4\left(\frac{\Gamma}{4}\right)^{\frac{2-\gamma_{1}}{4}} \varrho^{1-\frac{\left(2-\gamma_{1}\right)(n+2)}{4}}\right) \leq \omega\left(\Gamma \varrho^{\gamma_{1} \frac{n+2}{4}-\frac{n}{2}}\right)
$$


Note that the restriction $\gamma_{1}>\frac{2 n}{n+2}$ ensures that $\gamma_{1} \frac{n+2}{4}-\frac{n}{2}>0$. Summarized, we have $\omega\left(d_{\mathcal{P}}\left(z_{1}, z_{2}\right)\right) \leq \omega\left(\Gamma \varrho^{\alpha}\right)$ for all $z_{1}, z_{2} \in Q_{\varrho}^{(\lambda)}\left(z_{0}\right)$. Notice, that the dependence upon $p(\cdot)$ is continuously. Therefore, we can also replace dependence upon $p_{0}$ by a dependence on $\gamma_{1}$ and $\gamma_{2}$. Now, we divide the last estimate by $\left|Q_{\varrho}^{(\lambda)}\left(z_{0}\right)\right|$ and use Hölder's inequality with exponents $\frac{1}{1+\varepsilon_{1}}$ and $\frac{\varepsilon_{1}}{1+\varepsilon_{1}}$, thus we gain the following

$$
\begin{aligned}
& \mu f_{Q_{\varrho}^{(\lambda)}\left(z_{0}\right)}\left(s^{2}+|D v|^{2}+|D w|^{2}\right)^{\frac{p_{0}-2}{2}}|D(v-w)|^{2} \mathrm{~d} z \leq\left(f_{Q_{\varrho}^{(\lambda)}\left(z_{0}\right)}|D(v-w)|^{p_{0}} \mathrm{~d} z\right)^{\frac{1}{p_{0}}} \\
& \times c \omega\left(\Gamma \varrho^{\alpha}\right)\left(f_{Q_{w \varrho}^{(\lambda)}\left(z_{0}\right)}\left[1+|D v|^{p_{0}^{\prime}(p(\cdot)-1)}\right] \mathrm{d} z+\left(f_{Q_{\varrho}^{(\lambda)}\left(z_{0}\right)}|D v|^{p_{0}\left(1+\varepsilon_{1}\right)} \mathrm{d} z\right)^{\frac{1}{1+\varepsilon_{1}}}\right. \\
& \left.+\int_{Q_{\varrho}^{(\lambda)}\left(z_{0}\right)}(1+|D v|)^{\left.p_{0}^{\prime}(p \cdot \cdot)-1\right)} \log ^{p_{0}^{\prime}}(1+|D v|) \mathrm{d} z+\int_{Q_{Q}^{(\lambda)}\left(z_{0}\right)}(1+|D v|)^{p_{0}} \log ^{p_{0}^{\prime}}(1+|D v|) \mathrm{d} z\right)^{\frac{p_{0}-1}{p_{0}}} .
\end{aligned}
$$

Next, we note that the monotonicity of the logarithm implies $\log (e+a b) \leq \log (e+a)+\log (e+b)$ for all $a, b \geq 0$. Since the logarithm is monotone increasing and by the last inequality, we can conclude that

$$
\begin{aligned}
\log (1+|D v|) & \leq \log \left(e+(1+|D v|)^{p_{0}^{\prime}\left(p_{2}-1\right)}\right)=\log \left(e+(1+|D v|)^{p_{0}^{\prime}\left(p_{2}-1\right)} \frac{\left([1+|D v|]^{p_{0}^{\prime}\left(p_{2}-1\right)}\right)_{Q_{\varrho}^{(\lambda)}\left(z_{0}\right)}}{\left([1+|D v|]_{0}^{p_{0}^{\prime}\left(p_{2}-1\right)}\right)_{Q_{\varrho}^{(\lambda)}\left(z_{0}\right)}}\right) \\
& \left.\leq \log \left(e+\frac{(1+|D v|)^{p_{0}^{\prime}\left(p_{2}-1\right)}}{\left([1+|D v|]^{\prime}\left(p_{2}-1\right)\right.}\right)_{Q_{\varrho}^{(\lambda)}\left(z_{0}\right)}\right)+\log \left(e+\left([1+|D v|]^{p_{0}^{\prime}\left(p_{2}-1\right)}\right)_{Q_{\varrho}^{(\lambda)}\left(z_{0}\right)}\right)
\end{aligned}
$$

and also that

$$
\log (1+|D v|) \leq \log \left(e+(1+|D v|)^{p_{0}}\right) \leq \log \left(e+\frac{(1+|D v|)^{p_{0}}}{\left([1+|D v|]^{p_{0}}\right)_{Q_{Q}^{(\lambda)}\left(z_{0}\right)}}\right)+\log \left(e+\left([1+|D v|]^{p_{0}}\right)_{Q_{Q}^{(\lambda)}\left(z_{0}\right)}\right)
$$

is in force. Combining the last three estimate, we derive at

$$
\begin{aligned}
& \mu f_{Q_{Q}^{(\lambda)}\left(z_{0}\right)}\left(s^{2}+|D v|^{2}+|D w|^{2}\right)^{\frac{p_{0}-2}{2}}|D(v-w)|^{2} \mathrm{~d} z \leq\left(f_{Q_{Q}^{(\lambda)}\left(z_{0}\right)}|D(v-w)|^{p_{0}} \mathrm{~d} z\right)^{\frac{1}{p_{0}}} \\
& \times c \omega\left(\Gamma \varrho^{\alpha}\right)\left[f_{Q_{\varrho}^{(\lambda)}\left(z_{0}\right)}\left[1+|D v|^{p_{0}^{\prime}\left(p_{2}-1\right)}\right] \mathrm{d} z+\left(f_{Q_{\varrho}^{(\lambda)}\left(z_{0}\right)}|D v|^{p_{0}\left(1+\varepsilon_{1}\right)} \mathrm{d} z\right)^{\frac{1}{1+\varepsilon_{1}}}\right. \\
& +\int_{Q_{\varrho}^{(\lambda)}\left(z_{0}\right)}(1+|D v|)^{p_{0}^{\prime}\left(p_{2}-1\right)} \log ^{p_{0}^{\prime}}\left(e+\frac{(1+|D v|)^{p_{0}^{\prime}\left(p_{2}-1\right)}}{\left([1+|D v|]^{p_{0}^{\prime}\left(p_{2}-1\right)}\right)_{Q_{Q}^{(\lambda)}\left(z_{0}\right)}}\right) \mathrm{d} z \\
& +f_{Q_{\varrho}^{(\lambda)}\left(z_{0}\right)}(1+|D v|)^{p_{0}^{\prime}\left(p_{2}-1\right)} \log ^{p_{0}^{\prime}}\left(e+\left([1+|D v|]^{p_{0}^{\prime}\left(p_{2}-1\right)}\right){ }_{Q_{\varrho}^{(\lambda)}\left(z_{0}\right)}\right) \mathrm{d} z \\
& +\int_{Q_{Q}^{(\lambda)}\left(z_{0}\right)}(1+|D v|)^{p_{0}} \log ^{p_{0}^{\prime}}\left(e+\frac{(1+|D v|)^{p_{0}}}{\left([1+|D v|]^{p_{0}}\right)_{Q_{Q}^{(\lambda)}\left(z_{0}\right)}}\right) \mathrm{d} z \\
& \left.+\int_{Q_{\varrho}^{(\lambda)}\left(z_{0}\right)}(1+|D v|)^{p_{0}} \log ^{p_{0}^{\prime}}\left(e+\left([1+|D v|]^{p_{0}}\right) Q_{Q}^{(\lambda)}\left(z_{0}\right)\right) \mathrm{d} z\right]^{\frac{p_{0}-1}{p_{0}}} \\
& =c \omega\left(\Gamma \varrho^{\alpha}\right)\left(f_{Q_{Q}^{(\lambda)}\left(z_{0}\right)}|D(v-w)|^{p_{0}} \mathrm{~d} z\right)^{\frac{1}{p_{0}}}(I+I I+I I I+I V+V+V I)^{\frac{p_{0}-1}{p_{0}}}
\end{aligned}
$$

with the obvious labeling of $I-V I$. Here, we want to estimate $I I I$ and $V$ by the inequality (16). Therefore, we have to choose on the one hand $\mathfrak{g}_{1}:=(1+|D v|)^{p_{0}^{\prime}\left(p_{2}-1\right)}$ and

$$
\varsigma_{1}:=\frac{1+\varepsilon_{1}}{1+\frac{\varepsilon}{\gamma_{1}}}=c\left(n, \gamma_{1}, \gamma_{1}, \mu, L, \sigma\right)>1
$$


and on the other hand $\mathfrak{g}_{2}:=(1+|D v|)^{p_{0}}$ and $\varsigma_{2}:=\left(1+\varepsilon_{1}\right)=c\left(n, \gamma_{1}, \gamma_{1}, \mu, L, \sigma\right)>1$ with respect to (40) and (42). Moreover, we choose $Q=Q_{\varrho}^{(\lambda)}\left(z_{0}\right)$. Thus, we get

$$
I I I \leq c\left(\varsigma_{1}, \gamma_{1}, \gamma_{2}\right)\left(f_{Q}\left|\mathfrak{g}_{1}\right|^{\varsigma_{1}} \mathrm{~d} z\right)^{\frac{1}{\varsigma_{1}}}
$$

and

$$
I V \leq c\left(\varsigma_{2}, \gamma_{1}, \gamma_{2}\right)\left(f_{Q}\left|\mathfrak{g}_{2}\right|^{\varsigma_{2}} \mathrm{~d} z\right)^{\frac{1}{\varsigma_{2}}} .
$$

Now, we want to use (42) to bound $I I I$ and $I V$. The term $I V$, we can immediately bound by (42), i.e

$$
I V \leq c\left(f_{Q_{Q}^{(\lambda)}\left(z_{0}\right)} 1+|D v|^{p_{0}\left(1+\varepsilon_{1}\right)} \mathrm{d} z\right)^{\frac{1}{1+\varepsilon_{1}}} \leq c\left(1+\lambda^{1+\varepsilon_{1}}\right)^{\frac{1}{1+\varepsilon_{1}}} \leq c \lambda
$$

with a constant $c=c\left(n, \gamma_{1}, \gamma_{1}, \mu, L, \sigma\right)$, since $\lambda \geq 1$. The term $I I I$, we can bound by (42) as follows. First, we use the Hölder's inequality. This yields

$$
\begin{aligned}
I I I & \leq c\left(f_{Q_{Q}^{(\lambda)}\left(z_{0}\right)}(1+|D v|)^{p_{0}\left(1+\frac{\varepsilon_{1}}{\gamma_{1}}\right) S_{1}} \mathrm{~d} z\right)^{\frac{1}{s_{1}} \cdot \frac{p_{2}-1}{\left(p_{0}-1\right)\left(1+\varepsilon_{1} / \gamma_{1}\right)}}=c\left(f_{Q_{Q}^{(\lambda)}\left(z_{0}\right)}(1+|D v|)^{p_{0}\left(1+\varepsilon_{1}\right)} \mathrm{d} z\right)^{\frac{\frac{1}{s_{1}} \cdot \frac{p_{2}-1}{\left(p_{0}-1\right)\left(1+\varepsilon_{1} / \gamma_{1}\right)}}{p_{2}-1}} \\
& \leq c\left(1+\lambda^{\frac{p_{2}-1}{p_{0}-1}}\right) \leq c \lambda^{\frac{p_{2}-1}{p_{0}-1}}=c \lambda^{1+\frac{p_{2}-p_{0}}{p_{0}-1}} \leq c \lambda
\end{aligned}
$$

with a constant $c=c\left(n, \gamma_{1}, \gamma_{1}, \mu, L, c_{*}, \hat{c}\right)$, where we used (42), $\lambda \geq 1$ and (39). Next, we estimate $I$ by (43). Thus, we have

$$
I \leq c(1+\lambda) \leq c \lambda
$$

with a constant $c=c\left(n, \gamma_{1}, \gamma_{1}, \mu, L, c_{*}, \hat{c}\right)$, where we again used $\lambda \geq 1$. The expression $I I$ can be also bounded by (42). This yields

$$
I I \leq c(1+\lambda) \leq c \lambda
$$

Furthermore, we consider the following

$$
\left([1+|D v|]^{p_{0}^{\prime}\left(p_{2}-1\right)}\right)_{Q_{\varrho}^{(\lambda)}\left(z_{0}\right)}=\int_{Q_{\varrho}^{(\lambda)}\left(z_{0}\right)}(1+|D v|)^{p_{0}^{\prime}\left(p_{2}-1\right)} \mathrm{d} z \leq 1+c \lambda \leq c \lambda \leq c\left(\frac{K H}{\varrho^{n+2}}\right)^{\frac{p_{0}}{2}}
$$

with a constant $c=c\left(n, \gamma_{1}, \gamma_{1}, \mu, L\right)$, where we utilized (43) and Lemma 7. This and (43) we apply to $I V$ to conclude that

$$
I V \leq \log ^{p_{0}^{\prime}}\left(e+c\left(\frac{K H}{\varrho^{n+2}}\right)^{\frac{p_{0}}{2}}\right) f_{Q_{\varrho}^{(\lambda)}\left(z_{0}\right)}(1+|D v|)^{p_{0}^{\prime}\left(p_{2}-1\right)} \mathrm{d} z \leq \log ^{p_{0}^{\prime}}\left(e+c\left(\frac{K H}{\varrho^{n+2}}\right)^{\frac{p_{0}}{2}}\right) \lambda
$$

with a constant $c=c\left(n, \gamma_{1}, \gamma_{1}, \mu, L\right)$. Note we can always assume $c\left(\frac{K H}{\varrho^{n+2}}\right)^{\frac{p_{0}}{2}} \geq e$ by possibly reducing the value of $\varrho_{3}$. This allows to deduce that

$$
\log ^{p_{0}^{\prime}}\left(e+c\left(\frac{K H}{\varrho^{n+2}}\right)^{\frac{p_{0}}{2}}\right) \leq \log ^{p_{0}^{\prime}}\left(c\left(\frac{K H}{\varrho^{n+2}}\right)^{\frac{p_{0}}{2}}\right)=\frac{p_{0}}{2} \log ^{p_{0}^{\prime}}\left(c \frac{K H}{\varrho^{n+2}}\right) \leq c H^{p_{0}^{\prime}} \log ^{p_{0}^{\prime}}\left(\frac{K}{\varrho^{n+2}}\right) \leq c H^{p_{0}^{\prime}} \log ^{p_{0}^{\prime}}\left(\frac{K}{\varrho}\right),
$$

where we also used the fact that $\log (c x) \leq c \log (x)$ for $c \geq 1$. Combining the last two estimate, we get

$$
I V \leq c H^{p_{0}^{\prime}} \log ^{p_{0}^{\prime}}\left(\frac{K}{\varrho}\right) \lambda
$$


with a constant $c=c\left(n, \gamma_{1}, \gamma_{1}, \mu, L\right)$. By the same arguments and (42), we can also conclude that

$$
V I \leq c H^{p_{0}^{\prime}} \log ^{p_{0}^{\prime}}\left(\frac{K}{\varrho}\right) \lambda
$$

with a constant $c=c\left(n, \gamma_{1}, \gamma_{1}, \mu, L\right)$. Finally, we had shown that

$$
\mu f_{Q_{\varrho}^{(\lambda)}\left(z_{0}\right)}\left(s^{2}+|D v|^{2}+|D w|^{2}\right)^{\frac{p_{0}-2}{2}}|D(v-w)|^{2} \mathrm{~d} z \leq\left(f_{Q_{\varrho}^{(\lambda)}\left(z_{0}\right)}|D(v-w)|^{p_{0}} \mathrm{~d} z\right)^{\frac{1}{p_{0}}} c \omega\left(\Gamma \varrho^{\alpha}\right) H \log \left(\frac{K}{\varrho}\right) \lambda^{\frac{p_{0}-1}{p_{0}}}
$$

with a constant $c=c\left(n, \gamma_{1}, \gamma_{1}, \mu, L, c_{*}, \hat{c}, \sigma\right)$. In the case $p_{0} \geq 2$, we get by the Young's inequality the following comparison estimate

$$
f_{Q_{\varrho}^{(\lambda)}\left(z_{0}\right)}|D(v-w)|^{p_{0}} \mathrm{~d} z \leq c \omega\left(\Gamma \varrho^{\alpha}\right)^{p_{0}^{\prime}} H^{p_{0}^{\prime}} \log ^{p_{0}^{\prime}}\left(\frac{K}{\varrho}\right) \lambda
$$

with a constant $c=c\left(n, \gamma_{1}, \gamma_{1}, \mu, L, c_{*}, \hat{c}, \sigma\right)$, while in the case $p_{0}<2$ we have to estimate the left-hand side from below as in the proof of Lemma (10). This yields

$$
\int_{Q_{\varrho}^{(\lambda)}\left(z_{0}\right)}|D(v-w)|^{p_{0}} \mathrm{~d} z \leq \tilde{\kappa} f_{Q_{\varrho}^{(\lambda)}\left(z_{0}\right)}|D v|^{p_{0}} \mathrm{~d} z+c_{\tilde{\kappa}} \omega\left(\Gamma \varrho^{\alpha}\right)^{p_{0}^{\prime}} H^{p_{0}^{\prime}} \log ^{p_{0}^{\prime}}\left(\frac{K}{\varrho}\right) \lambda
$$

for any $\tilde{\kappa} \in(0,1]$ and with a constant $c_{\tilde{\kappa}}=c\left(\tilde{\kappa}, n, \gamma_{1}, \gamma_{1}, \mu, L, c_{*}, \hat{c}, \sigma\right)$. Using Hölder's inequality with exponents $\frac{1}{1+\varepsilon_{1}}, \frac{\varepsilon_{1}}{1+\varepsilon_{1}}$ and (42), then we derive the third comparison estimate

$$
\int_{Q_{\varrho}^{(\lambda)}\left(z_{0}\right)}|D(v-w)|^{p_{0}} \mathrm{~d} z \leq\left[c \tilde{\kappa}+c_{\tilde{\kappa}} \omega\left(\Gamma \varrho^{\alpha}\right) H^{p_{0}^{\prime}} \log ^{p_{0}^{\prime}}\left(\frac{K}{\varrho}\right)\right] \lambda
$$

for any number $\tilde{\kappa} \in(0,1)$ and with constants $c_{\tilde{\kappa}}=c\left(\tilde{\kappa}, n, \gamma_{1}, \gamma_{1}, \mu, L, c_{*}, \hat{c}, \sigma\right) \geq 1$ and $c=c\left(n, \gamma_{1}, \gamma_{1}, \mu, L\right) \geq 1$. Finally, we apply the fact $|D w|^{p_{0}} \leq 2^{p_{0}-1}\left(|D v|^{p_{0}}+|D v-D w|^{p_{0}}\right)$ and again Hölder's inequality, (42) and (46). This yields the energy estimate for $D w$, i.e.

$$
\int_{Q_{\varrho}^{(\lambda)}\left(z_{0}\right)}|D w|^{p_{0}} \mathrm{~d} z \leq c\left(\tilde{\kappa}+c_{\tilde{\kappa}} \omega\left(\Gamma \varrho^{\alpha}\right)^{p_{0}^{\prime}} H^{p_{0}^{\prime}} \log ^{p_{0}^{\prime}}\left(\frac{K}{\varrho}\right)\right) \lambda
$$

with a constant $c=c\left(n, \gamma_{1}, \gamma_{1}, \mu, L\right)$.

Step 4: Proof of the Lipschitz bound. Next, we want to derive a Lipschitz bound to Dw. Therefore, we choose $c_{*}=c \cdot \max \{1, \delta(\tilde{\kappa}, H, \varrho)\}$, where

$$
\delta(\tilde{\kappa}, H, \varrho):=\left(\tilde{\kappa}+\frac{c_{\tilde{\kappa}}}{H}+c_{\tilde{\kappa}} \omega\left(\Gamma \varrho^{\alpha}\right)^{p_{0}^{\prime}} H^{p_{0}^{\prime}} \log ^{p_{0}^{\prime}}\left(\frac{K}{\varrho}\right)\right) .
$$

Here, we have to mention that we need the dependency of $\delta$ on the term $\frac{c_{\widetilde{K}}}{H}$ later for the comparison estimate between $D u$ and $D w$, which we derive in the next and final step. The next arguments are also true without $\frac{c_{\tilde{K}}}{H}$. Therefore, this implies the desired energy estimate

$$
\int_{Q_{\varrho}^{(\lambda)}\left(z_{0}\right)}|D w|^{p_{0}} \mathrm{~d} z \leq c_{*} \lambda
$$

Hence, we can apply Theorem 9 which implies the following Lipschitz bound

$$
\sup _{Q_{\frac{1}{2} \varrho}^{(\lambda)}\left(z_{0}\right)}|D w| \leq c_{\operatorname{Lip}} \lambda^{\frac{1}{p_{0}}}
$$

with a constant $c_{\text {Lip }}=c\left(n, \mu, L, p_{0}, c_{*}\right)$. Since, the dependence upon $p_{0}$ is continuous it can be replaced by a lager constant depending on $\gamma_{1}$ and $\gamma_{2}$ instead of $p_{0}$, i.e. $c_{\text {Lip }}=c\left(n, \gamma_{1}, \gamma_{2}, \mu, L, c_{*}\right)$. 
Step 5: Proof of the final comparison estimate. The last step is to derive a comparison estimate between Du and $D w$. Therefore, we consider the following

$$
\begin{aligned}
f_{Q_{\frac{1}{2} \varrho}^{(\lambda)}\left(z_{0}\right)}|D v-D w|^{p(\cdot)} \mathrm{d} z & =\int_{Q_{\frac{1}{2} \varrho}^{(\lambda)}\left(z_{0}\right)}|D v-D w|^{\frac{p_{0}}{2}}|D v-D w|^{p(\cdot)-\frac{p_{0}}{2}} \mathrm{~d} z \\
& \leq\left(\int_{Q_{\frac{1}{2} \varrho}^{(\lambda)}\left(z_{0}\right)}|D v-D w|^{p_{0}} \mathrm{~d} z\right)^{\frac{1}{2}}\left(f_{Q_{\frac{1}{2} \varrho}^{(\lambda)}\left(z_{0}\right)}|D v-D w|^{2 p(\cdot)-p_{0}} \mathrm{~d} z\right)^{\frac{1}{2}} \\
& \leq c \delta(\tilde{\kappa}, H, \varrho)^{\frac{1}{2}} \lambda^{\frac{1}{2}}\left(\int_{Q_{\frac{1}{2} \varrho}^{(\lambda)}\left(z_{0}\right)}|D v|^{2 p(\cdot)-p_{0}}+|D w|^{2 p(\cdot)-p_{0}} \mathrm{~d} z\right)^{\frac{1}{2}},
\end{aligned}
$$

where we used the Hölder's inequality and (46) with (48). Next, we observe the exponent $2 p(\cdot)-p_{0}$. This exponent can be estimated from above as follows

$$
2 p(\cdot)-p_{0} \leq p(\cdot)\left(1+\omega\left(\tilde{\Gamma}(2 \varrho)^{\alpha}\right)\right) \leq p(\cdot)\left(1+\omega\left(\tilde{\Gamma}\left(2 \varrho_{3}\right)^{\alpha}\right)\right) \leq p(\cdot)\left(1+\varepsilon_{0}\right),
$$

where we used (41). Using the preceding result, the Hölder's inequality, (39), (40) and $\lambda \geq 1$, we can conclude that

$$
\begin{aligned}
\int_{Q_{\frac{1}{2} \varrho}^{(\lambda)}\left(z_{0}\right)}|D v|^{2 p(\cdot)-p_{0}} \mathrm{~d} z & \leq 2^{n+2} \int_{Q_{\varrho}^{(\lambda)}\left(z_{0}\right)}|D v|^{p(\cdot)\left(1+\omega\left(\tilde{\Gamma}(2 \varrho)^{\alpha}\right)\right)} \mathrm{d} z+1 \leq 2^{n+2}\left(f_{Q_{\varrho}^{(\lambda)}\left(z_{0}\right)}|D v|^{p(\cdot)\left(1+\varepsilon_{0}\right)} \mathrm{d} z\right)^{\frac{1+\omega\left(\tilde{\Gamma}(2)^{\alpha}\right)}{1+\varepsilon_{0}}}+1 \\
& \leq c \lambda^{1+\omega\left(\tilde{\Gamma}(2 \varrho)^{\alpha}\right)}+1 \leq c \lambda,
\end{aligned}
$$

with a constant $c=\left(n, \gamma_{1}, \gamma_{2}, \mu, L\right)$, where we used (39). Thus we have the following estimate

$$
\int_{Q_{\frac{1}{2} \varrho}^{(\lambda)}\left(z_{0}\right)}|D v-D w|^{p(\cdot)} \mathrm{d} z \leq c \delta(\tilde{\kappa}, H, \varrho)^{\frac{1}{2}} \lambda^{\frac{1}{2}}\left(c \lambda+\int_{Q_{\frac{1}{2} \varrho}^{(\lambda)}\left(z_{0}\right)}|D w|^{2 p(\cdot)-p_{0}} \mathrm{~d} z\right)^{\frac{1}{2}} \leq c \delta(\tilde{\kappa}, H, \varrho)^{\frac{1}{2}} \lambda
$$

with a constant $c=c\left(n, \gamma_{1}, \gamma_{2}, \mu, L, p_{0}, \hat{c}\right)$, where we used (49), (39) and $\lambda \geq 1$ for the last estimate. Finally, we use the fact that $|D u-D w|^{p(\cdot)} \leq 2^{p(\cdot)-1}\left(|D u-D v|^{p(\cdot)}+|D v-D w|^{p(\cdot)}\right)$ and we can combine the preceding estimate with the first comparison estimate (36), then derive the final comparison estimate

$$
\int_{Q_{\frac{1}{2} \varrho}^{(\lambda)}\left(z_{0}\right)}|D u-D w|^{p(\cdot)} \mathrm{d} z \leq c \delta(\tilde{\kappa}, H, \varrho)^{\frac{1}{2}} \lambda
$$

for every radius $\varrho \in\left(0, \varrho_{3}\right)$ with a constant $c$, which depends on $n, \gamma_{1}, \gamma_{1}, \mu, L, L_{1}, \sigma, \kappa$ and $\hat{c}$ where $\varrho_{3}$ depends on $n, \gamma_{1}, \gamma_{2}, \mu, L, \kappa, K, H, \omega(\cdot)$ and $Q_{2 \varrho}^{(\lambda)}\left(z_{0}\right) \Subset \Omega_{T}$, satisfies the intrinsic relation (33).

\section{Proof of the Main Result}

Proof. The proof is divided into several steps.

Step 1: Choice of the intrinsic cylinders. Here, we will use the stopping time argument. Therefore, let $K \geq 1$ and suppose that (12) is valid. Then, we observe a standard parabolic cylinder $Q_{r} \equiv Q_{r}\left(\mathfrak{z}_{0}\right)$, such that $Q_{2 r} \Subset \Omega_{T}$. First, we define

$$
\lambda_{0}:=\left(f_{Q_{2 r}}|D u|^{p(\cdot)}+H\left(|F|^{p(\cdot)}+|f|^{\gamma_{1}^{\prime}}+1\right) \mathrm{d} z\right)^{d} \geq 1, \quad \text { where } d:=\sup _{Q_{2 r}} d(p(\cdot))
$$

and $d(\cdot)$ is defined in (14). Moreover, we consider the concentric parabolic cylinders $Q_{r} \subseteq Q_{r_{1}} \subset Q_{r_{2}} \subseteq Q_{2 r}$ for fixed radii $r \leq r_{1}<r_{2} \leq 2 r$, all the cylinders sharing the same center $\mathfrak{z}_{0}$. Next, we shall consider $\lambda$, which satisfies

$$
\lambda>B \lambda_{0}
$$


with

$$
B:=\left(\frac{8 \chi r}{r_{2}-r_{1}}\right)^{(n+2) d}
$$

where $\chi=\chi\left(n, L_{1}, \gamma_{1}\right) \geq 5$ denotes the corresponding constant from Lemma 5. In addition, we observe radii $s$, which are conform to

$$
\frac{1}{2 \chi} R_{0}=\frac{\min \left\{\lambda^{\frac{p_{0}-2}{2 p_{0}}}\right\}\left(r_{2}-r_{1}\right)}{3 \chi} \leq s \leq \frac{\min \left\{\lambda^{\frac{p_{0}-2}{2 p_{0}}}\right\}\left(r_{2}-r_{1}\right)}{2}=R_{0},
$$

where $p_{0}=p\left(z_{0}\right)$. Notice that the maximal radius $R_{0}$ is chosen, such that for all points $z_{0} \in Q_{r_{1}}$ and radii $s \leq R_{0}$ the inclusion $Q_{s}^{(\lambda)}\left(z_{0}\right) \subset Q_{r_{2}}$ is fulfilled. Next, we want to prove that for any $z_{0} \in Q_{r_{1}}$, there holds

$$
f_{Q_{s}^{(\lambda)}\left(z_{0}\right)}|D u|^{p(\cdot)}+H\left(|F|^{p(\cdot)}+|f|^{\gamma_{1}^{\prime}}+1\right) \mathrm{d} z<\lambda
$$

From this fact and the definition of $\lambda_{0}$ we can conclude that

$$
\begin{aligned}
f_{Q_{s}^{(\lambda)}\left(z_{0}\right)}|D u|^{p(\cdot)}+H\left(|F|^{p(\cdot)}+|f|^{\gamma_{1}^{\prime}}+1\right) \mathrm{d} z & \leq \frac{\left|Q_{2 r}\right|}{\left|Q_{s}^{(\lambda)}\left(z_{0}\right)\right|}\left(f_{Q_{2 r}}|D u|^{p(\cdot)}+H\left(|F|^{p(\cdot)}+|f|^{\gamma_{1}^{\prime}}+1\right) \mathrm{d} z\right) \\
& \leq\left(\frac{2 r}{s}\right)^{n+2} \lambda^{\frac{p_{0}-2}{p_{0}}} \lambda_{0}^{\frac{1}{d}}
\end{aligned}
$$

holds, where we used (51) for the last estimate. Next, we have to treat the two cases $2 \leq p_{0} \leq \gamma_{2}$ and $\gamma_{1} \leq p_{0}<2$ separated. In the case $p_{0} \geq 2$, we have $d\left(p_{0}\right)=\frac{p_{0}}{2}$ and $\min \left\{\lambda^{\frac{p_{0}-2}{2 p_{0}}}, 1\right\}=1$. Hence, this yields by (56), (54), (52) and (53) the following

$$
f_{Q_{s}^{(\lambda)}\left(z_{0}\right)}|D u|^{p(\cdot)}+H\left(|F|^{p(\cdot)}+|f|^{\gamma_{1}^{\prime}}+1\right) \mathrm{d} z \leq\left(\frac{2 r}{s}\right)^{n+2} \lambda^{\frac{p_{0}-2}{p_{0}}} \lambda_{0}^{\frac{1}{d}} \leq\left(\frac{8 \chi r}{r_{2}-r_{1}}\right)^{n+2} \lambda^{\frac{p_{0}-2}{p_{0}}} \lambda_{0}^{\frac{1}{d}}<\left(\frac{8 \chi r}{r_{2}-r_{1}}\right)^{n+2} \lambda^{\frac{p_{0}-2}{p_{0}}}\left(\frac{\lambda}{B}\right)^{\frac{1}{d}}=\lambda .
$$

In the case that $\gamma_{1} \leq p_{0}<2$, there is $d\left(p_{0}\right)=\frac{2 p_{0}}{p_{0}(n+2)-n}$ respectively $\frac{1}{d\left(p_{0}\right)}=\frac{n+2}{2}-\frac{n}{p_{0}}$ and $\min \left\{\lambda^{\frac{p_{0}-2}{2 p_{0}}}, 1\right\}=\lambda^{\frac{p_{0}-2}{2 p_{0}}}$, we can conclude, in the same way as in the preceding case, that

$$
f_{Q_{s}^{(\lambda)}\left(z_{0}\right)}|D u|^{p(\cdot)}+H\left(|F|^{p(\cdot)}+|f|^{\gamma_{1}^{\prime}}+1\right) \mathrm{d} z \stackrel{(54)}{\leq}\left(\frac{8 \chi r}{\lambda^{\frac{p_{0}-2}{p_{0}}}\left(r_{2}-r_{1}\right)}\right)^{n+2} \lambda^{\frac{p_{0}-2}{p_{0}}} \lambda_{0}^{\frac{1}{d}}<\left(\frac{8 \chi r}{r_{2}-r_{1}}\right)^{n+2} \frac{\lambda}{B^{\frac{1}{d}}}=\lambda,
$$

where we finally used (53). This addict the same estimate as in the case $2 \leq p_{0} \leq \gamma_{2}$. The availability can be shown easily. For the purpose, we consider the following calculation

$$
\left(\frac{1}{\lambda^{\frac{p_{0}-2}{2 p_{0}}}}\right)^{n+2} \lambda^{\frac{p_{0}-2}{p_{0}}} \lambda_{0}^{\frac{1}{d}}=\lambda^{\frac{n+2}{p_{0}}-\frac{n+2}{2}} \lambda^{\frac{p_{0}-2}{p_{0}}} \lambda_{0}^{\frac{1}{d}}<\lambda^{\frac{2}{p_{0}}-\frac{1}{d}} \lambda^{\frac{p_{0}-2}{p_{0}}} \lambda_{0}^{\frac{1}{d}}=\lambda\left(\frac{\lambda_{0}}{\lambda}\right)^{\frac{1}{d}}<\frac{\lambda}{B^{\frac{1}{d}}} .
$$

Thus, we proved (55).

In the following, we show that also a converse inequality holds true for small radii. Therefore, we consider parabolic cylinders of the type

$$
Q_{s}^{(\lambda)}\left(z_{0}\right) \quad \text { with } 0<s \leq \min \left\{\lambda^{\frac{p_{0}-2}{2 p_{0}}}, 1\right\}\left(\frac{r_{2}-r_{1}}{2}\right)=R_{0}
$$

are contained in $Q_{r_{2}}$, where $\lambda \geq \lambda_{0}$ and $z_{0} \in Q_{r_{1}}$. By the Lebesgue's differentiation theorem, we can conclude that for a.e. points $z_{0} \in Q_{r_{1}}$, which satisfy the condition $\left|D u\left(z_{0}\right)\right|^{p_{0}}>\lambda$, we have the following estimate

$$
\lim _{s \downarrow 0}\left(\oint_{Q_{s}^{(\lambda)}\left(z_{0}\right)}|D u|^{p(\cdot)}+H\left(|F|^{p(\cdot)}+|f|^{\gamma_{1}^{\prime}}+1\right) \mathrm{d} z\right) \geq\left|D u\left(z_{0}\right)\right|^{p_{0}}>\lambda .
$$


This can shown by the following fact: We know that in the Lebesgue points $z_{0}$ of $D u$ we have

$$
\lim _{s \downarrow 0} \int_{Q_{s}\left(z_{0}\right)}|D u|^{p(\cdot)} \mathrm{d} z=\left|D u\left(z_{0}\right)\right|^{p_{0}},
$$

see e.g. (Harjulehto \& Hästö, 2004). At this stage, it is worth to mention that the averages are taken with respect to the usual cylinders while in (57) we need to have the averages on cylinders $Q_{s}^{(\lambda)}\left(z_{0}\right)$. Since,

$$
Q_{s}^{(\lambda)}\left(z_{0}\right) \subseteq Q_{\mu s}\left(z_{0}\right) \text { for } \mu:=\max \left\{\lambda^{\frac{2-p_{0}}{2 p_{0}}}, 1\right\},
$$

we get

$$
\left.\lim _{s \downarrow 0} f_{Q_{s}^{(\lambda)}\left(z_{0}\right)}|| D u\right|^{p(\cdot)}-\left|D u\left(z_{0}\right)\right|^{p_{0}}\left|\mathrm{~d} z \leq \frac{\mu^{n+2}}{\lambda^{\frac{2-p_{0}}{2 p_{0}}}} \lim _{s \downarrow 0} f_{Q_{\mu s}\left(z_{0}\right)}\right||D u|^{p(\cdot)}-\left|D u\left(z_{0}\right)\right|^{p_{0}} \mid \mathrm{d} z=0 .
$$

Hence (57) is valid.

Now, we have on the one hand a cylinder $Q_{s}^{(\lambda)}\left(z_{0}\right)$, on which the integral is smaller than $\lambda$ and on the other hand we have shown that the integral over this cylinder is bigger than $\lambda$, see (57). More precisely, by the absolute continuity of the integral, we can conclude from (57) and (55) that, there exists a maximal radius

$$
0<\varrho_{z_{0}}<\min \left\{\lambda^{\frac{p_{0}-2}{2 p_{0}}}, 1\right\} \frac{\left(r_{2}-r_{1}\right)}{3 \chi},
$$

such that

$$
f_{Q_{Q_{\Omega_{0}}\left(z_{0}\right)}^{(\lambda)}}|D u|^{p(\cdot)}+H\left(|F|^{p(\cdot)}+|f|^{\gamma_{1}^{\prime}}+1\right) \mathrm{d} z=\lambda
$$

while for any

$$
s \in\left(\varrho_{z_{0}}, \min \left\{\lambda^{\frac{p_{0}-2}{2 p_{0}}}, 1\right\} \frac{\left(r_{2}-r_{1}\right)}{2}\right)
$$

we have the following estimate

$$
f_{Q_{s}^{(\lambda)}\left(z_{0}\right)}|D u|^{p(\cdot)}+H\left(|F|^{p(\cdot)}+|f|^{\gamma_{1}^{\prime}}+1\right) \mathrm{d} z<\lambda
$$

With the choice of $\varrho_{z_{0}}$, we define concentric parabolic cylinders centered in $z_{0} \in E\left(\lambda, r_{1}\right)$, where

$$
E\left(r_{1}, \lambda\right):=\left\{z \in Q_{r_{1}}: z \text { is a Lebesgue point of }|D u| \text { and }|D u(z)|^{p(z)} \geq \lambda\right\},
$$

as follows:

$$
Q_{z_{0}}^{0}:=Q_{\varrho_{z_{0}}}^{(\lambda)}, Q_{z_{0}}^{1}:=Q_{\chi \varrho_{z_{0}}}^{(\lambda)}, Q_{z_{0}}^{2}:=Q_{2 \chi \varrho_{z_{0}}}^{(\lambda)}, Q_{z_{0}}^{3}:=Q_{4 \chi \varrho_{z_{0}}}^{(\lambda)} .
$$

Then, we have $Q_{z_{0}}^{0} \subset Q_{z_{0}}^{1} \subset Q_{z_{0}}^{2} \subset Q_{z_{0}}^{3} \subset Q_{r_{2}}$ and for $j \in\{0, \ldots, 3\}$, there holds

$$
\frac{\lambda}{(4 \chi)^{n+2}} f_{Q_{z_{0}}^{j}}|D u|^{p(\cdot)}+H\left(|F|^{p(\cdot)}+|f|^{\gamma_{1}^{\prime}}+1\right) \mathrm{d} z+1 \leq \lambda .
$$

The upper bound follows from (59) and the maximal choice of the stopping radius $\varrho_{z 0}$, while the lower bound follows from (59) by enlarging the domain of the integration from $Q_{z_{0}}^{0}$ to $Q_{z_{0}}^{j}$ and taking into account that $\frac{\left|Q_{z_{0}}^{j}\right|}{\left|Q_{z_{0}}^{0}\right|} \leq$ $(4 \chi)^{n+2}$.

Step 2: Estimates on intrinsic cylinders. Here, we want to apply the comparison estimates from the previous section. Therefore, we fix one particular cylinder $Q_{z_{0}}^{0}$ and the comparison functions $v$ and $w$ as the unique solutions 
to the initial-boundary value problems (34) and (35) with $Q_{z_{0}}^{3}$ and $Q_{z_{0}}^{2}$ instead of $Q_{2 \varrho}^{(\lambda)}\left(z_{0}\right)$ and $Q_{\varrho}^{(\lambda)}\left(z_{0}\right)$. Due to (62) we know that (33) is valid with the choice $\kappa=\kappa\left(n, \gamma_{1}\right)=(4 \chi)^{n+2}$. Further, we suppose that

$$
r \leq r_{0} \leq \varrho_{3},
$$

where $\varrho_{3}=\varrho_{3}\left(n, \gamma_{1}, \gamma_{2}, \mu, L, K, H, \omega(\cdot)\right) \in(0,1)$ denotes the radius introduced in (38) for the choice $\kappa=\kappa\left(n, \gamma_{1}\right)=$ $(4 \chi)^{n+2}$. Hence, we are allowed to apply (36), (49) and (50) with $\kappa=\kappa\left(n, \gamma_{1}\right)=(4 \chi)^{n+2}$ for any radius smaller than $\varrho_{3}$. Thus, from (49) used with $\kappa=\kappa\left(n, \gamma_{1}\right)=(4 \chi)^{n+2}$, we can conclude that

$$
\sup _{Q_{z_{0}}^{1}}|D w| \leq c_{\text {Lip }} \lambda^{\frac{1}{p_{0}}},
$$

where $c_{\text {Lip }}=c\left(n, \gamma_{1}, \gamma_{2}, \mu, L, \delta(\cdot)\right) \geq 1$, where $\delta(\cdot)$ is defined in (48). Next, we use the fact that

$$
|D u|^{p(\cdot)} \leq 2^{p(\cdot)-1}\left(|D w|^{p(\cdot)}+|D u-D w|^{p(\cdot)}\right) \leq c_{\mathrm{Lip}}^{p(\cdot)} \lambda^{\frac{p(\cdot)}{p_{0}}}+c\left(\gamma_{2}\right)|D u-D w|^{p(\cdot)} \quad \text { on } Q_{z 0}^{1} .
$$

Then, we integrate the previous estimate by $z$ over $Q_{z_{0}}^{1} \cap E\left(r_{1}, \mathcal{A} \lambda\right)$, where we had chosen

$$
\mathcal{A} \geq c_{\text {Lip }}^{\gamma_{2}} e^{\frac{3 n}{\alpha}} \geq c_{\text {Lip }}^{p(\cdot)} \lambda^{\frac{p(\cdot)}{p_{0}}} \geq 1
$$

where $c_{\text {Lip }}=c\left(n, \gamma_{1}, \gamma_{2}, \mu, L, c_{*}\right) \geq 1$. Then, (63), (64), (65), $2 \mathcal{A} \lambda<|D u|^{p(\cdot)}$ on $E\left(r_{1}, 2 \mathcal{A} \lambda\right)$ and the fact that

$$
|D w|^{p(\cdot)} \leq c_{\text {Lip }}^{p(\cdot)} \lambda^{\frac{p(\cdot)}{p_{0}}} \leq c_{\text {Lip }}^{p(\cdot)}\left(\frac{|D u|^{p(\cdot)}}{2 \mathcal{A}}\right) \lambda^{\frac{p(\cdot)-p_{0}}{p_{0}}} \leq \frac{c_{\text {Lip }}^{\gamma_{2}}}{2 \mathcal{A}} e^{\frac{3 n}{\alpha}}|D u|^{p(\cdot)} \leq \frac{1}{2}|D u|^{p(\cdot)}
$$

[cf. (18) and (65)] yields

$$
\int_{Q_{z_{0}}^{1} \cap E\left(r_{1}, 2 \mathcal{A} \lambda\right)}|D u|^{p(\cdot)} \mathrm{d} z \leq \frac{1}{2} \int_{Q_{z_{0}}^{1} \cap E\left(r_{1}, 2 \mathcal{A} \lambda\right)}|D u|^{p(\cdot)} \mathrm{d} z+c\left(\gamma_{2}\right) \int_{Q_{z_{0}}^{1} \cap E\left(r_{1}, 2 \mathcal{A} \lambda\right)}|D u-D w|^{p(\cdot)} \mathrm{d} z .
$$

Moreover, we apply the third comparison estimate from the preceding section, i.e. (50) with $\kappa=\kappa\left(n, \gamma_{1}\right)=(4 \chi)^{n+2}$, where we replace the cylinder $Q_{\frac{1}{2} \varrho}^{(\lambda)}\left(z_{0}\right)$ by $Q_{z_{0}}^{1}$ and (63) to derive the following energy estimate

$$
\int_{Q_{z_{0}}^{1} \cap E\left(r_{1}, 2 \mathcal{A} \lambda\right)}|D u|^{p(\cdot)} \mathrm{d} z \leq c \delta(\tilde{\kappa}, H, \varrho)^{\frac{1}{2}} \lambda\left|Q_{z_{0}}^{1}\right|
$$

with a constant $c=c\left(n, \gamma_{1}, \gamma_{2}, \mu, L, \hat{c}, c_{*}\right)$. Moreover, we recall that this estimate holds for any $\lambda>B \lambda_{0}$ and $z_{0} \in E\left(r_{1}, \lambda\right)$. Next, we will infer a bound for the measure of the cylinders $Q_{z_{0}}^{0}$. Note that, (59) implies

$$
\left|Q_{z_{0}}^{0}\right|=\frac{1}{\lambda} \int_{Q_{z_{0}}^{0}}|D u|^{p(\cdot)} \mathrm{d} z+\frac{1}{\lambda} \int_{Q_{z_{0}}^{0}} H\left(|F|^{p(\cdot)}+|f|^{\gamma_{1}^{\prime}}+1\right) \mathrm{d} z .
$$

Then, we split the first integral of the preceding estimate as follows

$$
\int_{Q_{z_{0}}^{0}}|D u|^{p(\cdot)} \mathrm{d} z=\int_{Q_{z_{0}}^{0} \cap\left\{|D u|^{p(\cdot)} \leq \lambda / 4\right\}}|D u|^{p(\cdot)} \mathrm{d} z+\int_{Q_{z_{0}}^{0} \cap E\left(r_{2}, \lambda / 4\right)}|D u|^{p(\cdot)} \mathrm{d} z \leq \frac{\lambda}{4}\left|Q_{z_{0}}^{0}\right|+\int_{Q_{z_{0}}^{0} \cap E\left(r_{2}, \lambda / 4\right)}|D u|^{p(\cdot)} \mathrm{d} z,
$$

and similarly the second one

$$
\int_{Q_{z_{0}}^{0}} H\left(|F|^{p(\cdot)}+|f|^{\gamma_{1}^{\prime}}+1\right) \mathrm{d} z \leq \frac{\lambda}{4}\left|Q_{z_{0}}^{0}\right|+\int_{Q_{z_{0}^{0}}^{0}\left\{\left\{H \left(|F|^{p(\cdot)}+|f|^{\left.\left.\gamma_{1}^{\prime}+1\right)>\lambda / 4\right\}}\right.\right.\right.} H\left(|F|^{p(\cdot)}+|f|^{\gamma_{1}^{\prime}}+1\right) \mathrm{d} z .
$$

Finally, we combine the last three estimate. This implies

$$
\left|Q_{z 0}^{0}\right| \leq \frac{2}{\lambda} \int_{Q_{z_{0}}^{0} \cap E\left(r_{2}, \lambda / 4\right)}|D u|^{p(\cdot)} \mathrm{d} z+\frac{2}{\lambda} \int_{Q_{20}^{0} \cap\left\{H\left(|F|^{p(\cdot)}+|f|^{\left.\gamma_{1}^{\prime}+1\right)>\lambda / 4}\right\}\right.} H\left(|F|^{p(\cdot)}+|f|^{\gamma_{1}^{\prime}}+1\right) \mathrm{d} z .
$$


Therefore, we have from (66) the following estimate

$$
\begin{aligned}
\int_{Q_{z_{0}}^{1} \cap E\left(r_{1}, 2 \mathcal{A} \lambda\right)}|D u|^{p(\cdot)} \mathrm{d} z & \leq c \delta(\tilde{\kappa}, H, \varrho)^{\frac{1}{2}} \frac{\left|Q_{z_{0}}^{1}\right|}{\left|Q_{z_{0}}^{0}\right|}\left(\int_{Q_{z_{0}}^{0} \cap E\left(r_{2}, \lambda / 4\right)}|D u|^{p(\cdot)} \mathrm{d} z\right. \\
& \left.+\int_{Q_{z_{0}}^{0} \cap\left\{H \left(|F|^{p(\cdot)}+|f|^{\gamma_{1}^{\prime}}\right.\right.} H(\mid)>\lambda / 4\right\}
\end{aligned}
$$

with a constant $c=c\left(n, \gamma_{1}, \gamma_{2}, \mu, L, \hat{c}, c_{*}\right)$.

Step 3: Estimates on the level sets. In the following, we will use the notation

$$
E(\varrho, \lambda):=\left\{z \in Q_{\varrho}:|D u|^{p(z)}>\lambda\right\} \quad \text { and } G(\varrho, \lambda):=\left\{z \in Q_{\varrho}: H\left(|F|^{p(\cdot)}+|f|^{\gamma_{1}^{\prime}}+1\right)>\lambda\right\}
$$

for the upper level sets of $|D u|^{p(\cdot)}$ and $\left(|F|^{p(\cdot)}+|f|^{\gamma_{1}^{\prime}}+1\right)$ on cylinders $Q_{\varrho}$ with $\varrho \in[r, 2 r]$. For $\eta \in(0,1)$, which we have to fix later, we consider the level sets $E(\varrho, \eta \lambda)$ and $G(\varrho, \eta \lambda)$. Our next aim, is to extend the last estimate. Therefore, we have to apply a covering argument. First, we construct a suitable covering of $E\left(r_{1}, \lambda\right)$ by intrinsic cylinders of the type as in (61). Note that, we have shown in the previous steps, that there exists a radius $\varrho_{z_{0}}$, which satisfies (58), such that on the cylinders $Q_{z_{0}}^{j}, j \in\{0, \ldots, 3\}$ the estimates (62) and (68) hold. Next, we want to apply the Vitali-type covering argument from Lemma 5. For this aim, we note that (59) and (18) with $\kappa=1$ imply, that

$$
\lambda \leq\left(\frac{\beta_{n} H K}{\varrho_{z 0}^{n+2}}\right)^{\frac{p_{0}}{2}}
$$

Therefore, (15) is valid for the family $\mathcal{F}:=\left\{Q_{z_{0}}^{0}\right\}$ of parabolic cylinders with center $z_{0} \in E\left(r_{1}, \lambda\right)$ (note that by possibly reducing the value of $r_{0}$, we can ensure that $\varrho_{z_{0}} \leq r \leq r_{0} \leq \varrho_{0}$ ). From the Vitali-type covering Lemma, we can conclude the existence of a countable subfamily $\left\{Q_{z_{i}}^{0}\right\}_{i=1}^{\infty} \subset \mathcal{F}$ of pairwise disjoint parabolic cylinders, such that the $\chi$-times enlarged cylinders $Q_{z_{i}}^{1}$ cover the set $E\left(r_{1}, \mathcal{A} \lambda\right)$, i.e. $E\left(r_{1}, 2 \mathcal{A} \lambda\right) \subset E\left(r_{1}, \lambda\right) \subset \bigcup_{i=1}^{\infty} Q_{z_{i}}^{1}$. In addition, there holds $Q_{z_{i}}^{3} \subset Q_{r_{2}}$. Moreover, we can conclude

$$
\begin{aligned}
& \int_{E\left(r_{1}, 2 \mathcal{A} \lambda\right)}|D u|^{p(\cdot)} \mathrm{d} z \leq \sum_{i=1}^{\infty} \int_{Q_{z_{i}}^{1}}|D u|^{p(\cdot)} \mathrm{d} z=\sum_{i=1}^{\infty} \int_{Q_{z_{i}}^{1} \cap E\left(r_{1}, 2 \mathcal{A} \lambda\right)}|D u|^{p(\cdot)} \mathrm{d} z+\sum_{i=1}^{\infty} \int_{Q_{z_{i}}^{1} \backslash E\left(r_{1}, 2 \mathcal{A} \lambda\right)}|D u|^{p(\cdot)} \mathrm{d} z \\
& \leq c \delta(\tilde{\kappa}, H, \varrho)^{\frac{1}{2}} \sum_{i=1}^{\infty}\left(\int_{Q_{z_{i}}^{0} \cap E\left(r_{2}, \lambda \backslash 4\right)}|D u|^{p(\cdot)} \mathrm{d} z+\int_{Q_{z_{i}}^{0} \cap\left\{H \left(|F|^{p(\cdot)}+|f|^{\left.\left.\gamma_{1}^{\prime}+1\right)>\lambda \backslash 4\right\}}\right.\right.} H\left(|F|^{p(\cdot)}+|f|^{\gamma_{1}^{\prime}}+1\right) \mathrm{d} z\right) \\
& +\int_{\bigcup_{i=1}^{\infty} Q_{z_{i}}^{1} \backslash E\left(r_{1}, 2 \mathcal{A} \lambda\right)}|D u|^{p(\cdot)} \mathrm{d} z \\
& \leq c \delta(\tilde{\kappa}, H, \varrho)^{\frac{1}{2}}\left(\int_{E\left(r_{2}, \lambda / 4\right)}|D u|^{p(\cdot)} \mathrm{d} z+\int_{G\left(r_{2}, \lambda / 4\right)} H\left(|F|^{p(\cdot)}+|f|^{\gamma_{1}^{\prime}}+1\right) \mathrm{d} z\right)
\end{aligned}
$$

with a constant $c=c\left(n, \gamma_{1}, \gamma_{2}, \mu, L, \hat{c}, c_{*}\right)$, where we used $\bigcup_{i=1}^{\infty} Q_{z_{i}}^{1} \backslash E\left(r_{1}, 2 \mathcal{A} \lambda\right) \neq \emptyset$ and (68). Notice that, the estimate (69) holds for every Lebesgue point $z_{0} \in Q_{r_{1}}$ of $D u$ with $\left|D u\left(z_{0}\right)\right|>\lambda>B \lambda_{0}$.

Step 4: The final estimate. We begin by defining truncations

$$
T_{k}:[0, \infty) \rightarrow[0, k], \quad T_{k}(\sigma):=\min \{\sigma, k\}
$$

and

$$
E_{k}\left(r_{1}, 2 \mathcal{A} \lambda\right):=\left\{z \in Q_{r_{1}}: T_{k}\left(|D u(z)|^{p(z)}\right)>2 \mathcal{A} \lambda\right\}
$$

In order to derive integrability estimates for $D u$ from the above estimate (69) on the super-level sets, we will employ Fubini's Theorem. Starting point is (69) with respect to the truncations, i.e. for $k \geq B \lambda_{0}$

$$
\int_{E_{k}\left(r_{1}, 2 \mathcal{A} \lambda\right)}|D u|^{p(\cdot)} \mathrm{d} z \leq c \delta(\tilde{\kappa}, H, \rho)^{\frac{1}{2}}\left(\int_{E_{k}\left(r_{2}, \lambda / 4\right)}|D u|^{p(\cdot)} \mathrm{d} z+\int_{G\left(r_{2}, \lambda / 4\right)} H\left(|F|^{p(\cdot)}+|f|^{\gamma_{1}^{\prime}}+1\right) \mathrm{d} z\right) .
$$


Here, we distinguish the cases $k \leq 2 \mathcal{A} \lambda$ and $k>2 \mathcal{A} \lambda$. The case $k \leq 2 \mathcal{A} \lambda$ implies $E_{k}\left(r_{1}, 2 \mathcal{A} \lambda\right)=\emptyset$ and therefore, the estimate (70) holds, since

$$
0 \leq c \delta(\tilde{\kappa}, H, \rho)^{\frac{1}{2}}\left(\int_{E_{k}\left(r_{2}, \lambda / 8\right)}|D u|^{p(\cdot)} \mathrm{d} z+\int_{G\left(r_{2}, \lambda / 4\right)} H\left(|F|^{p(\cdot)}+|f|^{\gamma_{1}^{\prime}}+1\right) \mathrm{d} z\right) .
$$

In the case $k>2 \mathcal{A} \lambda$ the estimate (70) holds, since $E_{k}\left(r_{1}, 2 \mathcal{A} \lambda\right)=E\left(r_{1}, 2 \mathcal{A} \lambda\right)$ and $E_{k}\left(r_{2}, \frac{\lambda}{4}\right)=E\left(r_{2}, \frac{\lambda}{4}\right)$, cf. (69). Now, we multiply both side of (70) by $\lambda^{q-2}$. Then, we integrate with respect to $\lambda$ over $\left(B \lambda_{0}, \infty\right)$. This yields

$$
\begin{aligned}
\int_{B \lambda_{0}}^{\infty} \lambda^{q-2} \int_{E_{k}\left(r_{1}, 2 \mathcal{A} \lambda\right)}|D u|^{p(\cdot)} \mathrm{d} z \mathrm{~d} \lambda & \leq c \delta(\tilde{\kappa}, H, \rho)^{\frac{1}{2}}\left[\int_{B \lambda_{0}}^{\infty} \lambda^{q-2} \int_{E_{k}\left(r_{2}, \lambda / 4\right)}|D u|^{p(\cdot)} \mathrm{d} z \mathrm{~d} \lambda\right. \\
& \left.+c \int_{B \lambda_{0}}^{\infty} \lambda^{q-2} \int_{G\left(r_{2}, \lambda / 8\right)} H\left(|F|^{p(\cdot)}+|f|^{\gamma_{1}^{\prime}}+1\right) \mathrm{d} z \mathrm{~d} \lambda\right] .
\end{aligned}
$$

Next, we use Fubini's Theorem as follows

$$
\begin{aligned}
\int_{B \lambda_{0}}^{\infty} \lambda^{q-2} \int_{E_{k}\left(r_{1}, 2 \mathcal{A} \lambda\right)}|D u|^{p(\cdot)} \mathrm{d} z \mathrm{~d} \lambda & =\int_{E_{k}\left(r_{1}, 2 \mathcal{A} B \lambda_{0}\right)}|D u|^{p(\cdot)} \int_{B \lambda_{0}}^{T_{k}\left(|D u|^{p \cdot(\cdot) / 2 \mathcal{A}}\right.} \lambda^{q-2} \mathrm{~d} \lambda \mathrm{d} z \\
& =\frac{(2 \mathcal{A})^{1-q}}{(q-1)} \int_{E_{k}\left(r_{1}, 2 \mathcal{A} B \lambda_{0}\right)}|D u|^{p(\cdot)} T_{k}\left(|D u|^{p(\cdot)}\right)^{q-1} \mathrm{~d} z \\
& -\frac{\left(B \lambda_{0}\right)^{q-1}}{q-1} \int_{E_{k}\left(r_{1}, 2 \mathcal{A} B \lambda_{0}\right)}|D u|^{p(\cdot)} \mathrm{d} z \\
& \geq \frac{(2 \mathcal{A})^{1-q}}{(q-1)} \int_{Q_{r_{1}}}|D u|^{p(\cdot)} T_{k}\left(|D u|^{p(\cdot)}\right)^{q-1} \mathrm{~d} z-\frac{\left(B \lambda_{0}\right)^{q-1}}{q-1} \int_{Q_{r_{1}}}|D u|^{p(\cdot)} \mathrm{d} z,
\end{aligned}
$$

where we used the facts $Q_{r_{1}}=E_{k}\left(r_{1}, 2 \mathcal{A} B \lambda_{0}\right) \cup\left(Q_{r_{1}} \backslash E_{k}\left(r_{1}, 2 \mathcal{A} B \lambda_{0}\right)\right)$ and

$$
T_{k}\left(|D u|^{p(\cdot)}\right) \leq 2 \mathcal{A B} \lambda_{0} \quad \text { on } \quad\left(Q_{r_{1}} \backslash E_{k}\left(r_{1}, 2 \mathcal{A} B \lambda_{0}\right)\right) .
$$

Again by Fubini's Theorem, we gain

$$
\begin{aligned}
\int_{B \lambda_{0}}^{\infty} \lambda^{q-2} \int_{E_{k}\left(r_{2}, \lambda / 8\right)}|D u|^{p(\cdot)} \mathrm{d} z \mathrm{~d} \lambda & =\int_{E_{k}\left(r_{2}, B \lambda_{0} / 4\right)}|D u|^{p(\cdot)} \int_{B \lambda_{0}}^{T_{k}\left(|D u|^{p(\cdot)}\right)} \lambda^{q-2} \mathrm{~d} \lambda \mathrm{d} z \\
& \leq \frac{4^{q-1}}{q-1} \int_{Q_{r_{2}}}|D u|^{p(\cdot)} T_{k}\left(|D u|^{p(\cdot)}\right)^{q-1} \mathrm{~d} z
\end{aligned}
$$

and

$$
\begin{aligned}
\int_{B \lambda_{0}}^{\infty} \lambda^{q-2} \int_{G\left(r_{2}, \lambda / 8\right)} H\left(|F|^{p(\cdot)}+|f|^{\gamma_{1}^{\prime}}+1\right) \mathrm{d} z \mathrm{~d} \lambda & =\int_{G\left(r_{2}, B \lambda_{0} / 8\right)} H\left(|F|^{p(\cdot)}+|f|^{\gamma_{1}^{\prime}}+1\right) \int_{B \lambda_{0}}^{4 H\left(|F|^{(\cdot)}+|f|^{\gamma_{1}^{\prime}}+1\right)} \lambda^{q-2} \mathrm{~d} \lambda \mathrm{d} z \\
& \leq \frac{4^{q-1} H^{q}}{q-1} \int_{Q_{r_{2}}}\left(|F|^{p(\cdot)}+|f|^{\gamma_{1}^{\prime}}+1\right)^{q} \mathrm{~d} z .
\end{aligned}
$$

Plugging the last three estimate into (71). This yields

$$
\begin{aligned}
\int_{Q_{r_{1}}}|D u|^{p(\cdot)} T_{k}\left(|D u|^{p(\cdot)}\right)^{q-1} \mathrm{~d} z & \leq\left(2 \mathcal{A} B \lambda_{0}\right)^{q-1} \int_{Q_{r_{1}}}|D u|^{p(\cdot)} \mathrm{d} z+c \mathcal{A}^{q-1} \delta(\tilde{\kappa}, H, \varrho)^{\frac{1}{2}} \int_{Q_{r_{2}}}|D u|^{p(\cdot)} T_{k}\left(|D u|^{p(\cdot)}\right)^{q-1} \mathrm{~d} z \\
& +c \mathcal{A}^{q-1} H^{q} \delta(\widetilde{\kappa}, H, \varrho)^{\frac{1}{2}} \int_{Q_{r_{2}}}\left(|F|^{p(\cdot)}+|f|^{\gamma_{1}^{\prime}}+1\right)^{q} \mathrm{~d} z
\end{aligned}
$$

with a constant $c=c\left(n, \gamma_{1}, \gamma_{2}, \mu, L, \hat{c}, c_{*}, q\right)$. Notice that the estimate stays stable as $q \downarrow 0$.

Step 5: Choice of parameters and adjusting the exponents. Here, we will mainly choose the parameter $H$ and finally the maximal radius $r_{0}$. The aim is to choose the parameter such that

$$
c_{q} \mathcal{A}^{q-1} \delta(\tilde{\kappa}, H, \varrho)^{\frac{1}{2}} \leq \vartheta \in(0,1),
$$


where $\mathcal{A} \geq c_{\text {Lip }}^{\gamma_{2}} e^{\frac{3 n}{\alpha}}$ and $\delta$ are introduced in (65) and (48), respectively, with constants $c_{\tilde{\kappa}}=c\left(\tilde{\kappa}, n, \gamma_{1}, \gamma_{2}, \mu, L\right)$ and $c=c\left(n, \gamma_{1}, \gamma_{2}, \mu, L, \sigma, \kappa\right)$. This we can achieve by first choosing $\tilde{\kappa} \in(0,1)$ sufficiently small, e.g. $c_{q} \mathcal{A}^{q-1} \tilde{\kappa}^{\frac{1}{2}} \leq \frac{\vartheta}{4}$. Then, we have to choose $H=H\left(n, \gamma_{1}, \gamma_{2}, q, \mu, L, \sigma, \kappa\right) \geq 1$ large enough, depending on the fixed value of $\tilde{\kappa}$, e.g. $c_{q} \mathcal{A}^{q-1}\left(\frac{c_{\tilde{\kappa}}}{H}\right)^{\frac{1}{2}} \leq \frac{\vartheta}{4}$. Finally, we have to reduce the value $\varrho_{0}$, now depending on $n, \gamma_{1}, \gamma_{2}, q, \mu, L, \sigma, \kappa, K, \omega(\cdot)$ small, such that for all $\varrho \in\left(0, r_{0}\right)$

$$
c_{q} \mathcal{A}^{q-1}\left[c_{\tilde{\kappa}} \omega\left(\Gamma(2 \varrho)^{\alpha}\right) H^{p_{0}^{\prime}} \log ^{p_{0}^{\prime}}\left(\frac{K}{\varrho}\right)\right]^{\frac{1}{2}} \leq \frac{\vartheta}{2},
$$

where we used (8). Then, we gain from (72) the following

$$
\begin{aligned}
\int_{Q_{r_{1}}}|D u|^{p(\cdot)} T_{k}\left(|D u|^{p(\cdot)}\right)^{q-1} \mathrm{~d} z & \leq \vartheta \int_{Q_{r_{2}}}|D u|^{p(\cdot)} T_{k}\left(|D u|^{p(\cdot)}\right)^{q-1} \mathrm{~d} z+c\left(\frac{r}{r_{2}-r_{1}}\right)^{\beta} \lambda_{0}^{q-1} \int_{Q_{2 r}}|D u|^{p(\cdot)} \mathrm{d} z \\
& +c \int_{Q_{2 r}}\left(|F|^{p(\cdot)}+|f|^{\gamma_{1}^{\prime}}+1\right)^{q} \mathrm{~d} z .
\end{aligned}
$$

Now, we are in the situation to use the iteration Lemma 4, with the choices $t=r_{1}, s=r_{2}, \varrho=2 r$

$$
\phi(R) \equiv \int_{Q_{R}}|D u|^{p(\cdot)} T_{k}\left(|D u|^{p(\cdot)}\right)^{q-1} \mathrm{~d} z, \quad A \equiv c r^{\beta} \lambda_{0}^{q-1} \int_{Q_{2 r}}|D u|^{p(\cdot)} \mathrm{d} z \quad \text { and } \quad C \equiv c \int_{Q_{2 r}}\left(|F|^{p(\cdot)}+|f|^{\gamma_{1}^{\prime}}+1\right)^{q} \mathrm{~d} z .
$$

This yields

$$
\phi(r) \leq c(\beta, \vartheta)\left[\frac{A}{r^{\beta}}+C\right] .
$$

Finally, passing to the limit $k \rightarrow \infty$, which is possible by Fatou's Lemma and taking average, we find that

$$
f_{Q_{r}}|D u|^{p(\cdot) q} \mathrm{~d} z \leq c\left[\lambda_{0}^{q-1} f_{Q_{2 r}}|D u|^{p(\cdot)} \mathrm{d} z+f_{Q_{2 r}}\left(|F|^{p(\cdot)}+|f|^{\gamma_{1}^{\prime}}+1\right)^{q} \mathrm{~d} z\right] .
$$

Note that $c=c\left(n, \gamma_{1}, \gamma_{2}, q, \mu, L, \sigma, \kappa, K, \omega(\cdot)\right)$, since $\beta$ depends continuously on $p(\cdot)$, i.e. the dependence upon $p(\cdot)$ via the parameter $d$ can be replaced by a dependence on $\gamma_{1}$ and $\gamma_{2}$. Since, $Q_{2 r} \Subset \Omega_{T}$ was arbitrary, we proved

$$
|D u|^{p(\cdot)} \in L_{\mathrm{loc}}^{q}\left(\Omega_{T}\right) .
$$

Step 6: Adjusting the exponent. In the final step, it remains to show the estimate (13). First, we remember the choice of $\lambda_{0}$ in (51). Next, we plug $\lambda_{0}$ into (74). This yields

$$
f_{Q_{r}}|D u|^{p(\cdot) q} \mathrm{~d} z \leq c\left[\left(f_{Q_{2 r}}|D u|^{p(\cdot)}+H\left(|F|^{p(\cdot)}+|f|^{\gamma_{1}^{\prime}}+1\right) \mathrm{d} z\right)^{d(q-1)} f_{Q_{2 r}}|D u|^{p(\cdot)} \mathrm{d} z+f_{Q_{2 r}}\left(|F|^{p(\cdot)}+|f|^{\gamma_{1}^{\prime}}+1\right)^{q} \mathrm{~d} z\right]
$$

with a constant $c$ depending on $n, \gamma_{1}, \gamma_{2}, q, \mu, L, \sigma, \kappa, \omega(\cdot)$. Next, we use Hölder's inequality as follows

$$
f_{Q_{2 r}} H\left(|F|^{p(\cdot)}+|f|^{\gamma_{1}^{\prime}}+1\right) \mathrm{d} z \leq \frac{1}{\left|Q_{2 r}\right|}\left(\int_{Q_{2 r}} H^{q^{\prime}} \mathrm{d} z\right)^{\frac{1}{q^{\prime}}}\left(\int_{Q_{2 r}}\left(|F|^{p(\cdot)}+|f|^{\gamma_{1}^{\prime}}+1\right)^{q} \mathrm{~d} z\right)^{\frac{1}{q}}=H\left(f_{Q_{2 r}}\left(|F|^{p(\cdot)}+|f|^{\gamma_{1}^{\prime}}+1\right)^{q} \mathrm{~d} z\right)^{\frac{1}{q}} .
$$

Combining the last two estimates, this yields

$$
\begin{aligned}
f_{Q_{r}}|D u|^{p(\cdot) q} \mathrm{~d} z & \leq c H\left(f_{Q_{2 r}}|D u|^{p(\cdot)}+\left(f_{Q_{2 r}}\left(|F|^{p(\cdot)}+|f|^{\gamma_{1}^{\prime}}+1\right)^{q} \mathrm{~d} z\right)^{\frac{1}{q}}\right)^{1+d(q-1)}+c f_{Q_{2 r}}\left(|F|^{p(\cdot)}+|f|^{\gamma_{1}^{\prime}}+1\right)^{q} \mathrm{~d} z \\
& \leq c H\left(f_{Q_{2 r}}|D u|^{p(\cdot)}+\left(f_{Q_{2 r}}\left(|F|^{p(\cdot)}+|f|^{\gamma_{1}^{\prime}}+1\right)^{q} \mathrm{~d} z\right)^{\frac{1}{q}}\right)^{1+d(q-1)}+c\left(f_{Q_{2 r}}\left(|F|^{p(\cdot)}+|f|^{\gamma_{1}^{\prime}}+1\right)^{q} \mathrm{~d} z\right)^{\frac{1}{q}(1+d(q-1))} \\
& \leq c H\left(f_{Q_{2 r}}|D u|^{p(\cdot)}+\left(f_{Q_{2 r}}\left(|F|^{p(\cdot)}+|f|^{\gamma_{1}^{\prime}}+1\right)^{q} \mathrm{~d} z\right)^{\frac{1}{q}}\right)^{1+d(q-1)},
\end{aligned}
$$


i.e. a version of the Calderón-Zygmund estimate (13) only for $d:=\sup _{Q_{2 r}} d(p(\cdot))$. But our aim is to show (13) with $d\left(p_{0}\right)$ instead of $d$, where $d\left(p_{0}\right)$ is defined in (14) with $p_{0}=p\left(\mathfrak{z}_{0}\right)$ and $\mathfrak{z}_{0}$ is the center of the cylinder $Q_{2 r} \equiv Q_{2 r}\left(\mathfrak{z}_{0}\right) \Subset \Omega_{T}$. Therefore, we consider again the choice of $\lambda_{0}$ from (51) as follows

$$
\begin{aligned}
\lambda_{0}=\left(f_{Q_{2 r}}|D u|^{p(\cdot)}+H\left(|F|^{p(\cdot)}+|f|^{\gamma_{1}^{\prime}}+1\right) \mathrm{d} z\right)^{d} & =\left(f_{Q_{2 r}}|D u|^{p(\cdot)}+H\left(|F|^{p(\cdot)}+|f|^{\gamma_{1}^{\prime}}+1\right) \mathrm{d} z\right)^{d-d\left(p_{0}\right)} \\
& \times\left(f_{Q_{2 r}}|D u|^{p(\cdot)}+H\left(|F|^{p(\cdot)}+|f|^{\gamma_{1}^{\prime}}+1\right) \mathrm{d} z\right)^{d\left(p_{0}\right)}
\end{aligned}
$$

where $p_{0}=p\left(\mathfrak{z}_{0}\right)$. Here, it is obvious that we derive to (13) if we can show that

$$
\left(f_{Q_{2 r}}|D u|^{p(\cdot)}+H\left(|F|^{p(\cdot)}+|f|^{\gamma_{1}^{\prime}}+1\right) d z\right)^{d-d\left(p_{0}\right)} \leq(K(H+1))^{d-d\left(p_{0}\right)} \leq c,
$$

where we used (12) and therefore, we gain

$$
\lambda_{0} \leq c\left(f_{Q_{2 r}}|D u|^{p(\cdot)}+H\left(|F|^{p(\cdot)}+|f|^{\gamma_{1}^{\prime}}+1\right) \mathrm{d} z\right)^{d\left(p_{0}\right)} .
$$

To this aim, we first deduce an upper bound for $d-d\left(p_{0}\right)$ by a localization argument in terms of $\omega(r)$. Since, $d(p(\cdot))$ is continuous, there exists $\bar{z} \in \bar{Q}_{r}$, such that $d=d(p(\bar{z}))$. From the definition of $d(\cdot)$ in (14), we observe that

$$
d\left(p_{0}\right) \geq \max \left\{\frac{p_{0}}{2}, \frac{2 p_{0}}{p_{0}(n+2)-2 n}\right\} .
$$

In the case $p(\bar{z}) \geq 2$, we can conclude that

$$
d-d\left(p_{0}\right)=\frac{p(\bar{z})}{2}-d\left(p_{0}\right) \leq \frac{p(\bar{z})}{2}-\frac{p_{0}}{2}=\frac{1}{2}\left(p(\bar{z})-p_{0}\right) \leq \frac{1}{2} \omega(r),
$$

where we used (6). While in the case $p(\bar{z})<2$, we can conclude that

$$
d-d\left(p_{0}\right) \leq \frac{2 p(\bar{z})}{p(\bar{z})(n+2)-2 n}-\frac{2 p_{0}}{p_{0}(n+2)-2 n} \leq \frac{4 n}{\left[\gamma_{1}(n+2)-2 n\right]^{2}} \omega(r),
$$

where we used $p(\bar{z}) \leq p_{0}$ and again (6). Thus, from the last two estimate, we gain an upper bound for $d-d\left(p_{0}\right)$, i.e. $d-d\left(p_{0}\right) \leq c\left(n, \gamma_{1}\right) \omega(r)$. This bound we apply to

$$
\begin{aligned}
\left(f_{Q_{2 r}}|D u|^{p(\cdot)}+H\left(|F|^{p(\cdot)}+|f|^{\gamma_{1}^{\prime}}+1\right) \mathrm{d} z\right)^{d-d\left(p_{0}\right)} & \leq\left(f_{Q_{2 r}}|D u|^{p(\cdot)}+H\left(|F|^{p(\cdot)}+|f|^{\gamma_{1}^{\prime}}+1\right) \mathrm{d} z\right)^{c\left(n, \gamma_{1}\right) \omega(r)} \\
& \leq\left(r^{-(n+2)} K(H+1)\right)^{c\left(n, \gamma_{1}\right) \omega(r)} .
\end{aligned}
$$

From (8), we have $r^{-\omega(r)} \leq e$. This implies

$$
\left(f_{Q_{2 r}}|D u|^{p(\cdot)}+H\left(|F|^{p(\cdot)}+|f|^{\gamma_{1}^{\prime}}+1\right) \mathrm{d} z\right)^{d-d\left(p_{0}\right)} \leq e^{(n+2) c\left(n, \gamma_{1}\right)}(K(H+1))^{c\left(n, \gamma_{1}\right) \omega(r)}=e^{c\left(n, \gamma_{1}\right)}(K(H+1))^{c\left(n, \gamma_{1}\right) \omega(r)},
$$

where we used the fact that $K^{c\left(n, \gamma_{1}\right) \omega(r)}=e^{c\left(n, \gamma_{1}\right) \omega(r) \log K} \leq e^{c\left(n, \gamma_{1}\right) \omega(r) \log \frac{1}{r}} \leq e^{c\left(n, \gamma_{1}\right)}$, cf. (8). Notice, that we determined $H=H\left(n, \gamma_{1}, \gamma_{2}, q, \mu, L, \sigma, \kappa\right) \geq 1$ in Step 5 of this proof. Therefore, we have

$$
\left(f_{Q_{2 r}}|D u|^{p(\cdot)}+H\left(|F|^{p(\cdot)}+|f|^{\gamma_{1}^{\prime}}+1\right) \mathrm{d} z\right)^{d-d\left(p_{0}\right)} \leq c(K H)^{\omega(r)} .
$$

with a constant $c=c\left(n, \gamma_{1}, \gamma_{2}, \mu, L, q\right)$. Finally, we use again (8) to estimate the last factor on the right-hand side, i.e.

$$
(K H)^{\omega(r)} \leq \exp [\omega(r) \log (K H)] \leq \exp \left[\omega(r) \log \left(\frac{1}{r}\right)\right] \leq e,
$$

provided $r \leq r_{0} \leq \min \{\rho, 1 /(K H)\}$, where $\rho \in(0,1]$ is the radius from (8). This yields (13). Thus, we have completed the proof of Theorem 3. Using these argument, we can infer from (74) the Calderón-Zygmund estimate (13) from Theorem 3. 


\section{Acknowledgements}

The author would like to thank Verena Bögelein for many interesting discussions during his PhD study about regularity theory for parabolic problems. Moreover, the author is very thankful to Christoph Scheven for many fruitful discussions about parabolic obstacle problem.

\section{References}

Acerbi, E., \& Mingione, G. (2001). Regularity Results for a Class of Functionals with Non-Standard Growth. Arch. Rational Mech. Anal., 156, 121-140. http://dx.doi.org/10.1007/s002050100117

Acerbi, E., \& Mingione, G. (2002a). Regularity Results for Stationary Electro-Rheological Fluids. Arch. Rational Mech. Anal., 164, 213-259. http://dx.doi.org/10.1007/s00205-002-0208-7

Acerbi, E., \& Mingione, G. (2002b). Regularity results for electrorheological fluids: the stationary case. C. R. Acad. Sci. Paris, Ser. I, 334(1), 817-822. http://dx.doi.org/10.1016/S1631-073X(02)02337-3

Acerbi, E., \& Mingione, G. (2005). Gradient estimates for the $p(x)$-Laplacean system. J. Reine Angew. Math., 584, 117-148. http://dx.doi.org/10.1515/crll.2005.2005.584.117

Acerbi, E., \& Mingione, G. (2007). Gradient estimates for a class of parabolic systems. Duke Math., 136(2), 285-320. http://dx.doi.org/10.1215/S0012-7094-07-13623-8

Acerbi, E., Mingione, G., \& Seregin G.A. (2004). Regularity results for parabolic systems related to a class of non-Newtonian fluids. Annales de l'Institut Henri Poincare (C) Non Linear Analysis, 21(1), 25-60. http://dx.doi.org/10.1016/j.anihpc.2002.11.002

Antontsev, S.N., \& Shmarev, S.I. (2005). A model porous medium equation with variable exponent of nonlinearity: existence, uniqueness and localization properties of solutions. Nonlinear Anal., 60(3), 515-545. http://dx.doi.org/10.1016/j.na.2004.09.026

Baroni, P., \& Habermann, J. (2012). Calderón-Zygmund estimates for parabolic measure data equations. J. Differ. Equ., 252(1), 412-447. http://dx.doi.org/10.1016/j.jde.2011.08.016

Baroni, P. (2013). New contributions to nonlinear Calderón-Zygmund theory. PhD Thesis, Scuola Normale Superiore. http://cvgmt.sns.it/paper/2508/

Baroni, P., \& Bögelein, V. (2013). Calderón-Zygmund estimates for parabolic $p(x, t)$-Laplacian systems. (Rev. Mat. Iberoam, to appear). http://cvgmt.sns.it/paper/2212/

Bögelein, V. (2013). Global Calderón-Zygmund theory for nonlinear parabolic systems. Calc. Var. Partial Differential Equations, to appear. http://dx.doi.org/10.1007/s00526-013-0687-4

Bögelein, V., \& Duzaar, F. (2011). Higher integrability for parabolic systems with non-standard growth and degenerate diffusions. Publ. Mat., 55(1), 201-250. http://dx.doi.org/10.5565/PUBLMAT_55111_10

Bögelein, V., \& Duzaar, F. (2012). Hölder estimates for parabolic $p(x, t)$-Laplacian systems. Mathematische Annalen, 354(3), 907-938. http://dx.doi.org/10.1007/s00208-011-0750-4

Bögelein, V., Duzaar, F., \& Mingione, G. (2011). Degenerate problems with irregular obstacles. J. Reine Angew. Math., 650, 107-160. http://dx.doi.org/10.1515/CRELLE.2011.006

Caffarelli, L., \& Peral, I. (1998). On $W^{1, p}$ estimates for elliptic equations in divergence from. Comm. Pure Appl. Math., 51, 1-21.

DiBenedetto, E. (1993). Degenerate Parabolic Equations. Universitext. Springer-Verlag, New York.

DiBenedetto, E., \& Friedman, A. (1984). Regularity of solutions of nonlinear degenerate parabolic systems. J. Reine Angew. Math., 349, 83-128. http://dx.doi.org/10.1515/crll.1984.349.83

DiBenedetto, E., \& Friedman, A. (1985a). Hölder estimates for nonlinear degenerate parabolic sytems. J. Reine Angew. Math., 357, 1-22. http://dx.doi.org/10.1515/crll.1985.357.1

DiBenedetto, E., \& Friedman, A. (1985b). Addendum to "Hölder estimates for nonlinear degenerate parabolic Systems". J. Reine Angew. Math., 363, 217-220. http://dx.doi.org/10.1515/crll.1985.363.217

DiBenedetto, E., \& Manfredi, J. (1993). On the higher integrability of the gradient of weak solutions of certain degenerate elliptic systems. Am. J. Math.(5), 1107-1134. 
Duzaar, F., Mingione, G., \& Steffen, K. (2011). Parabolic systems with polynomial growth and regularity. Mem. Am. Math. Soc., 214(1005). http://dx.doi.org/10.1090/S0065-9266-2011-00614-3

Eleuteri, M., \& Habermann, J. (2010). Calderón-Zygmund type estimates for a class of obstacle problems with $p(x)$-growth. J. Math. Anal. Appl., 372, 140-161. http://dx.doi.org/10.1016/j.jmaa.2010.05.072

Erhardt, A. (2013a). Existence and Gradient Estimates in Parabolic Obstacle Problems with Nonstandard Growth. PhD Thesis. University of Erlangen-Nuremberg.

Erhardt, A. (2013b). Existence of soultions to parabolic problems with nonstandard growth and irregular obstacle. Preprint, submitted. http://cvgmt.sns.it/paper/2350/

Erhardt, A. (2013c). Higher integrability for soultions to parabolic problems with irregular obstacle and nonstandard growth. Preprint, submitted. http://cvgmt.sns.it/paper/2351/

Erhardt, A. (2013d). Hölder estimates for parabolic obstacle problems. Ann. Mat. Pura Appl. (4), to appear. http://dx.doi.org/10.1007/s10231-013-0392-0

Erhardt, A. (2014). Calderón-Zygmund theory for parabolic obstacle problems with nonstandard growth. Adv. Nonlinear Anal, 3(1), 15-44. http://dx.doi.org/10.1515/anona-2013-0024

Ettwein, F., \& Růǔička, M. (2003). Existence of Strong Solutions for Electrorheological Fluids in Two Dimensions: Steady Dirichlet Problem. Geometric Analysis and Nonlinear Partial Differential Equations, 591-602. http://dx.doi.org/10.1007/978-3-642-55627-2_31

Giaquinta, M. (1983). Multiple Integrals in the Calculus of Variations and Nonlinear Elliptic Systems. Princeton University Press, Princeton, New Jersey.

Habermann, J. (2008). Calderón-Zygmund estimates for higher order systems with $p(x)$-growth. Math. Z., 258, 427-462. http://dx.doi.org/10.1007/s00209-007-0180-x

Harjulehto, P., \& Hästö, P. (2004). Lebesgue points in variable exponent spaces, Ann. Acad. Sci. Fenn. Math., 29, 295-306. http://www.math.helsinki.fi/Annales/Vol29/harjul.html

Iwaniec, T. (1983). Projections onto gradient fields and $L^{p}$-estimates for degenerated elliptic operators. Stud. Math., 75, 293-312.

Iwaniec, T., \& Verde, A. (1999). On the Operator $\mathcal{L}(f)=f \log |f|$. J. Funct. Anal., 169(2), 391-420. http://dx.doi.org/10.1006/jfan.1999.3443

Kinnunen, J., \& Zhou, S. (1999). A Local estimate for nonlinear equations with discontinuous coefficients. Commun. Partial Differential Equation, 24(11-12), 2043-2068. http://dx.doi.org/10.1080/03605309908821494

Kinnunen, J., \& Lewis, J.L. (2000). Higher integrability for parabolic systems of p-Laplacian type. Duke Math. J., 102(2), 253-271. http://dx.doi.org/10.1215/S0012-7094-00-10223-2

Misawa, M. (2005). $L^{q}$ estimates of gradients for evolutional p-Laplacian systems. J. Differ. Equations, 219(2), 390-420. http://dx.doi.org/10.1016/j.jde.2004.11.003

Mingione, G. (2007a). Calderón-Zygmund estimates for measure data problems. C. R. Acad. Sci. Paris, Ser. I, 344(7), 437-442. http://dx.doi.org/10.1016/j.crma.2007.02.005

Mingione, G. (2007b).The Calderón-Zygmund theory for elliptic problems with measure data. Ann. Scuola Norm. Sup. Pisa Cl. Sci. (4), VI(2), 195-261. http://dx.doi.org/10.2422/2036-2145.2007.2.01

Mingione, G. (2010). Nonlinear Aspects of Calderón-Zygmund Theory. J. Deutsch. Math. Ver., 112(3), $159-191$. http://dx.doi.org/10.1365/s13291-010-0004-5

Růǔička, M. (2000). Electrorheological Fluids: Modeling and Mathematical Theory. Lecture Notes in Mathematics, 1748, Spinger-Verlag, Heidelberg.

Růǔička, M. (2004). Modeling, Mathematical and Numerical Analysis of Electrorheological Fluids. Appl. Math., 49(6), 565-609. http://dx.doi.org/10.1007/s10492-004-6432-8

Scheven, Ch. (2010). Nonlinear Calderón-Zygmund theory for parabolic systems with subquadratic growth. $J$. Evol. Equ., 10(3), 597-622. http://dx.doi.org/10.1007/s00028-010-0063-1

Scheven, Ch. (2011). Existence and Gradient Estimates in Nonlinear Problems with Irregular Obstacles. Habilita- 
tion Thesis, University Erlangen-Nuremberg.

Scheven, Ch. (2014). Existence of localizable solutions to nonlinear parabolic problems with irregular obstacles. Manuscripta Math, to appear. http://dx.doi.org/10.1007/s00229-014-0684-8

\section{Copyrights}

Copyright for this article is retained by the author(s), with first publication rights granted to the journal.

This is an open-access article distributed under the terms and conditions of the Creative Commons Attribution license (http://creativecommons.org/licenses/by/3.0/). 